\title{
Almost classical solutions to the total variation flow
}

\author{
Karolina Kielak, Piotr BogusŁaw Mucha and Piotr RybKa
}

\begin{abstract}
The paper examines the one-dimensional total variation flow equation with Dirichlet boundary conditions. Thanks to a new concept of "almost classical" solutions we are able to determine evolution of facets - flat regions of solutions. A key element of our approach is the natural regularity determined by the nonlinear elliptic operator, for which $x^{2}$ is an example of an irregular function. Such a point of view allows us to construct solutions. We apply this idea to numerical simulations for typical initial data. Due to the nature of Dirichlet data, any monotone function is an equilibrium. We prove that each solution reaches such a steady state in finite time.
\end{abstract}

\section{Introduction}

The equation which is the topic of this paper

$$
u_{t}-\frac{d}{\mathrm{~d} x}\left(\operatorname{sgn}\left(u_{x}\right)\right)=0, \quad u(a)=a_{b}, \quad u(b)=a_{e}, \quad a_{b}, a_{e} \in \mathbb{R}
$$

is a one-dimensional example of the total variation flow. The motivation to study this problem is twofold: (a) image analysis, see [1,5,27,34]; (b) crystal growth problems, see $[4,25,32,36]$. There are different physically relevant models, where a similar to ours surface energy appears, but the corresponding evolutionary problem is not necessarily set up, see for example, [11].

Equation (1.1) may be interpreted as a steepest descent of the total variation, that is, we can write (1.1) as a gradient flow $u_{t} \in-\partial E(u)$ for a functional $E$. This is why we can apply the abstract nonlinear semigroup theory of Komura, see [10,15], to obtain existence of solutions. This has been performed by [25,20,27] and also by $[2,3,7,12,14,33]$. However, the generality of this tool does not permit to study fine points of solutions to (1.1).

Solutions to (1.1) enjoy interesting properties, Fukui and Giga, [20], have noticed that facets persist. By a facet we mean a flat part (i.e., affine) of the solution with zero slope. Zero is exactly the point of singularity of function $|\cdot|$. This is why the problem of facet evolution is not only nonlocal but highly anisotropic. Our equation (1.1) is at least formally parabolic of the second order. This is why we call the above behavior

Mathematics Subject Classification: 35K55, 35K67, 74N05, 94A08.

Keywords: Total variation flow, optimal regularity, structure of solutions, almost classical solutions, sudden directional diffusion. 
of solutions the sudden directional diffusion. However, even more dramatic effects of singular diffusion can be seen in the fourth order problems, see [21].

As we have already mentioned, certain properties of facets were established in [20], for example, their finite speed of propagation was calculated. What is missing is the description of the process how they merge and how they are created. In [30], we studied a problem similar to (1.1). We worked there with a simplification of the flow of a closed curve by the singular mean weighted curvature. We have shown existence of so-called almost classical solutions, that is, there is a finite number of time instances when the time derivative does not exist. However, the results of [30] indicate lack of efficiency of the methods used there. This fact is our motivation to rebuilt the theory from the very beginning. For this reason, we consider here the model system admitting effects of sudden directional diffusion. Hoping that our approach will be suitable for more general systems.

Our approach is as follows. We notice that the implicit time discretization leads to a series of Yosida approximations to the operator on the right-hand-side (r.h.s. for short) of (1.1). We study them quite precisely, because we consider variable time steps. As a result, we capture the moment when two facets merge. We do not perform any further special considerations. We want to see how the regularity of original solutions is transported via solvability of the Yosida approximation. Due to the one-dimensional character of the problem, we are able to obtain a result so good that it is of the maximal regularity character, what is rather expected for quasilinear parabolic systems. Let us underline that properly understood smoothness is the most important question connected to solvability of the original system. We modify standard regularity setting in order to capture all phenomena appearing in the system. As a result of our considerations, we come to the conclusion that the best smoothness we could expect for a solution $u$ that $u(\cdot, t)$ be a piecewise linear function, while $x^{2}$ is an example of an irregular function.

Our main goal is monitoring the evolution, as well creation, of the facets and a precise description of the regularity of solutions to (1.1). For this purpose, we apply methods, which are distinctively different from those in the literature. We develop ideas which appeared in our earlier works. A key point is a construction of a proper composition of two multivalued operators: the first one is sgn understood as a maximal monotone graph, the other one is $u_{x}$, which is defined only a.e. We leave aside the issue that in general this is a measure, not a function. This problem is resolved differently by the authors applying the semigroup approach, [5, 12,20,25] etc. We treat $u_{x}$ as a Clarke differential (see (2.1) and the text below this formula). Here, we show that this composition is helpful when: we construct solutions, see Theorem 3.1; and we discuss regularity of solutions, see Theorems 2.1 and 2.2.

On the other hand, there are two sorts of results available up to now to deal with (1.1): (1) the method based on the abstract semigroup theory, see for example, [5,20,25] and [12]. It is very general and elegant, it enables us to study the facet motion, but it does not capture all relevant information. The intrinsic difficulty associated with this 
method is the fact that the energy functional corresponding to (1.1) is not coercive, also see below Lemma 2.1 and the proof of Theorem 3.1. (2) the method based on the appropriate definition of the viscosity solution [22,23]. However, a different kind of problem was studied there. This is an active research field, see [26,37].

Our approach is based on the Yosida approximation, defined by as a solution of the resolvent problem

$$
\lambda u-\frac{d}{\mathrm{~d} x}\left(\operatorname{sgn}\left(u_{x}\right)\right)=\lambda v \quad \text { in }(a, b),\left.\quad(u-v)\right|_{\partial[a, b]}=0 .
$$

There are a couple of points to be made here. Firstly, we will construct $u$, a solution to (1.2), by very simple means; this is done in Sect. 3. This process resembles looking for a good notion of a weak solutions to a PDE. Since we came up with an integral equation, we will call its solutions mild ones, see formula (3.15). Secondly, (1.2) may be interpreted as an Euler-Lagrange equation for a nonstandard variational functional. Namely, we set

$$
\mathcal{J}(u)= \begin{cases}\int_{a}^{b}|D u| & \text { if } u \in D(\mathcal{J}) \equiv\left\{u \in B V[a, b], u(a)=a_{b}, u(b)=a_{e}\right\}, \\ +\infty & \text { if } L_{2}(a, b) \backslash D(\mathcal{J}),\end{cases}
$$

where $\int_{a}^{b}|D u|$ is the total variation of measure $D u$. We stress that we consider the space $B V$ over a closed interval. Then, (1.2) may be seen as

$$
v \in u+h \partial \mathcal{J}(u),
$$

where $\partial \mathcal{J}$ is the subdifferential of $\mathcal{J}$ and $h=\frac{1}{\lambda}$. The form of (1.4) allows us to look at (1.2) as at a semidiscretization in time of the system (1.1). Thanks to Lemma 3.3 we are allowed to split and add time steps $h$, obtaining this way a semigroup generating the solution to (1.1). These properties are applied in Sect. 5.

Thus, no matter which point of view we adopted, $u$ is given as the action of the nonlinear resolvent operator $R(\lambda, A)$ on $v$, that is,

$$
u=R(\lambda, A)(v) \equiv(\lambda+A)^{-1}(v), \quad \text { where } A=-\frac{\partial}{\partial x} \operatorname{sgn} \frac{\partial}{\partial x} .
$$

However, the notion of a mild solution to (1.2) does not permit us to interpret this equation easily. On the other hand, by convex analysis, we can see (1.2) as an inclusion (1.4).

The definition of the nonlinear resolvent operator leads to a detailed study of $\mathcal{J}$. The advantage of (1.2) is that it permits to monitor closely behavior of facets. It says that the regularity propagates. That is, if $v$ is such that $v_{x}$ belongs to the $B V$ space and the number of connected components of the properly understood set $\left\{x: v_{x}(x)=0\right\}$ is finite, then $u_{x}$ has the same property for sufficiently large $\lambda$. It is well-known that the nonlinear resolvent leads to Yosida approximation, which is the key object in the construction of the nonlinear semigroup in the Komura theory. Namely, we set

$$
A_{\lambda} u=\lambda(u-R(\lambda, A)(\lambda u)) .
$$


Our observation is that a maximal monotone multivalued operator like sgn taking values in $[-1,1]$ may be composed with a multifunction properly generalizing a function of bounded total variation. We shall describe here this composition denoted by $\bar{o}$, see Sect. 2. We introduced such an operation in [30], see also [31]. We also point to an essential difficulty here, which is the problem of composition of two multivalued operators. Even if both of them are maximal monotone, the result need not be monotone nor single valued. If the outer of the two operators we compose is a subdifferential, then we expect that the result is closely related to the minimal section of the subdifferential.

One of our main results says that $A_{\lambda} u$ defined by (1.5) indeed converges to $-\frac{\partial}{\partial x} \operatorname{sgn} \bar{o} u_{x}$. Moreover, we have an error estimate, see Theorem 3.1, formula (3.4). We recall that in if $A$ is maximal monotone and $u \in D(A)$, then the Yosida approximation $A_{\lambda} u$ converges to the minimal section $A^{o} u$ of $A$, see [15, Proposition 2.6 (iii)]. In this way we justify correctness of the new notion. Due to the "explicit" nature of o, we may better describe the regularity of solutions to (1.2).

Once we have constructed the Yosida approximation, we show existence of solution to the approximating problem $u_{t}^{\lambda}=-A_{\lambda}\left(u^{\lambda}\right)$ on short time intervals, where $u^{\lambda}\left(t_{0}\right)$ is given. This is done in Lemma 4.1. In fact, the method is close in spirit to the construction of the nonlinear semigroup, see [17]. Convergence of the approximate solutions is shown at the end of Sect. 4. Here, we use the Yosida approximation to capture the finite number of time instances when the solution $u(t)$ is just right differentiable with respect to time; otherwise, the derivative exists. The point is that we control the distance to the original problem (1.1), so that we monitor the time instances when facets merge.

Let us tell few words about the approach of proving our result. First, we define a space of admissible functions giving regularity of constructed solutions. Furthermore, we state main results together with an explanation of the meaning of almost classical solutions. In Sect. 3, we study the Yosida approximation for our system, concentrating on qualitative analysis of solutions. Proofs in this part are based on a direct construction which is possible due to the fine properties of chosen regularity. Subsequently, we prove the main results concerning existence and regularity. Finally, we study the asymptotics of solutions and present an example of an explicit solution. We conclude our paper with numerical simulations. They are based upon the semidiscretization. Since they present a series of time snapshots, these pictures contain only the round-off error. At each time step, there is no discretization error. The examples in Sect. 5 present the typical behavior, for which each solution becomes a monotone function in finite time.

\section{The composition $\bar{o}$ and the main result}

Our main goal is to present a new approach to solvability of systems of type (1.1). The total variation flow is a good example for such experiment, since we know precisely the solution. The first step is to define the basic regularity class of functions. 
For the sake of definiteness, but without loss of generality, we assume that the domain $[a, b]=[0,1]$.

DEFINITION 2.1. (cf. [38, Chapter 5]) We say that a real valued function $u$, defined over a closed interval $[0,1]$, belongs to $B V[0,1]$, provided that $\|D u\| \equiv \int_{0}^{1}|D u|<$ $\infty$, where $|D u|$ is the total variation of the measure $D u$. We recall that

$$
\|u\|_{B V[0,1]}=\|D u\|+\|u\|_{1} .
$$

Additionally, we treat $B V$ functions as multi-valued function. This is easy for functions which are derivatives, $u_{x} \in B V[0,1]$. This is very useful in the regularity study of solution to (1.1). Indeed, if $u$ and $u_{x}$ belong to $B V[0,1]$, then $u$ is Lipschitz continuous. Hence, $\frac{d^{+} u}{\mathrm{~d} x}$ and $\frac{d^{-} u}{\mathrm{~d} x}$ exist everywhere and they differ on at most countable set. Thus, we may set

$$
\partial_{x} u(s)=\left\{\tau u_{x}^{-}+(1-\tau) u_{x}^{+}: \tau \in[0,1]\right\} .
$$

Under our assumptions on $u$, the set $\partial_{x} u(x)$ is the Clarke differential of $u$ and equality holds in (2.1) due to [16, Section 2, Ex. 1]. If $u$ is convex, then $\partial_{x} u$ is the well-known subdifferential of $u$. As a result, if $u_{x} \in B V$, then for each $x_{0} \in(0,1)$, we have $\partial_{x} u\left(x_{0}\right)=\left[\lim _{x \rightarrow x_{0}^{-}} u_{x}(x), \lim _{x \rightarrow x_{0}^{+}} u_{x}(x)\right]_{o r}$, where $[a, b]_{o r}=[a, b]$ for $a \leq b$ and $[a, b]_{o r}=[b, a]$ for $b>a$.

However, the description of solutions as functions whose derivatives belong to $B V$ is not sufficient. We have to restrict our attention to its subclass. There is a need to control the facets, which we shall explain momentarily. A facet of $u, F$ is a closed, connected piece of graph of $u$ with zero slope, that is, $F=F\left(\xi^{-}, \xi^{+}\right)=\{(x, y)$ : $y=$ const $\left.=u\left(\left[\xi^{-}, \xi^{+}\right]\right), x \in\left[\xi^{-}, \xi^{+}\right]\right\}$, which is maximal with respect to inclusion of sets. The interval $\left[\xi^{-}, \xi^{+}\right]$will be called the set of parameters or preimage of facet $F$.

Let us recall that zero is the only point, where the absolute value, $|\cdot|$, the integrand in the definition of $\mathcal{J}$, fails to be differentiable. Thus, the special role of the zero slope and facets.

We shall also distinguish a subclass of facets. We shall say that a facet $F\left(\xi^{-}, \xi^{+}\right)$ has zero curvature, if and only if there is such $\epsilon>0$, that function $u$ restricted to $\left[\xi^{-}-\epsilon, \xi^{+}+\epsilon\right]$ is monotone. In the case the function under consideration is increasing, this means that $u\left(\xi^{-}-\epsilon\right)<u\left(\xi^{-}\right)=u\left(\xi^{+}\right)<u\left(\xi^{+}+\epsilon\right)$. We shall see that zero curvature facets do not move at all. There may be even an infinite number of them. They have no influence on the evolution of the system. For that reason, we introduce the following objects, capturing the essential phenomena. We shall say that a facet $F\left(\zeta^{-}, \zeta^{+}\right)$of $u$ is an essential facet and it will be denoted by $F_{e s s}\left(\zeta^{-}, \zeta^{+}\right)$, provided that there exists $\epsilon>0$ such that either

$u$ is decreasing on $\left(\zeta^{-}-\epsilon, \zeta^{-}\right)$and $u(t)>u\left(\zeta^{-}\right)$for $t \in\left(\zeta^{-}-\epsilon, \zeta^{-}\right)$ and $u$ is increasing on $\left(\zeta^{+}, \zeta^{+}+\epsilon\right)$ and $u(t)>u\left(\zeta^{+}\right)$for $t \in\left(\zeta^{+}, \zeta^{+}+\epsilon\right)$ (then we call such a facet convex); moreover, we set

$$
\operatorname{sgn} \kappa_{\left[\zeta^{-}, \zeta^{+}\right]}=1
$$


or

$u$ is increasing on $\left(\zeta^{-}-\epsilon, \zeta^{-}\right)$and $u(t)<u\left(\zeta^{-}\right)$for $t \in\left(\zeta^{-}-\epsilon, \zeta^{-}\right)$and $u$ is decreasing on $\left(\zeta^{+}, \zeta^{+}+\epsilon\right)$ and $u(t)<u\left(\zeta^{+}\right)$for $t \in\left(\zeta^{+}, \zeta^{+}+\epsilon\right)$ (then we call such facet concave); moreover, we set

$$
\operatorname{sgn} \kappa_{\left[\zeta^{-}, \zeta^{+}\right]}=-1
$$

It may happen that $\zeta^{-}=\zeta^{+}=: \zeta$, then we shall call $F(\zeta, \zeta)$ a degenerate essential facet. In this case $u$ has a strict local minimum or a strict maximum at point $\zeta$.

We will call $\operatorname{sgn} \kappa_{\left[\zeta^{-}, \zeta^{+}\right]}$the transition number of facet $F\left(\zeta^{-}, \zeta^{+}\right)$. For the sake of consistency, we set the transition number $\operatorname{sgn} \kappa_{\left[\zeta^{-}, \zeta^{+}\right]}$to zero for a zero curvature facet $F\left(\zeta^{-}, \zeta^{+}\right)$. In addition, if $\zeta^{-}=0$ or $\zeta^{+}=1$, then we set to zero the transition number of $F\left(\zeta^{-}, \zeta^{+}\right)$.

The union of parameter sets of all essential facets is denoted by $\Xi_{\text {ess }}(w)$ and $K_{\text {ess }}(w)$ is the number of essential facets, including degenerate facets.

DEFINITION 2.2. Let us suppose that $w=\partial_{x} u \in B V[0,1]$, where $u$ is absolutely continuous and $\partial_{x} u$ is the Clarke differential of $u$. Let $\Xi(w)=\{x \in[0,1]: 0 \in w(x)\}$. We say that $w$ as above is J-regular or shorter $w \in \mathrm{J}$-R iff the set $\Xi_{\text {ess }}(w) \subset \Xi(w)$ consists of a finite number of components, that is,

$$
\Xi_{e s s}(w)=\left[a_{1}, b_{1}\right] \cup \cdots \cup\left[a_{K_{\text {ess }}(w)}, b_{K_{\text {ess }}(w)}\right] \text { where } a_{i} \leq b_{i}
$$

and each interval $\left[a^{i}, b^{i}\right]$ is an argument set of an essential (nondegenerate or degenerate) facet $F\left(a^{i}, b^{i}\right)$. In particular, components of $\Xi(w) \backslash \Xi_{e s s}(w)$ consist only of arguments of zero curvature facets of $u$. Our definition in particular excludes functions with fast oscillations like $x^{2} \sin \frac{1}{x}$. We distinguished above a subset of $B V$ functions. Since degenerate facets will be treated as pathology, for given $w \in \mathrm{J}-\mathrm{R}$, we define

$$
L(w)=\min \left\{b-a:[a, b] \text { is a connected component of } \Xi_{e s s}(w)\right\} .
$$

Note that $L(w)=0$ iff there exists a degenerate facet of $u$.

The name J-regular refers to the regularity of the integrand in the functional $\mathcal{J}$, which has singular point at $p=0 . J$-regularity of $w=\partial u_{x}$ means that function $u$ can be split into finite number of subdomains where it is monotone. We also define the following quantity,

$$
\|w\|_{\mathrm{J}-\mathrm{R}[0,1]}=\|w\|_{B V[0,1]}+K_{\text {ess }}(w),
$$

where $K_{\text {ess }}(w)$ is the number of connected parts of $\Xi_{\text {ess }}(w)$; however, this is not a norm.

We start with the definition of a useful class of admissible functions.

DEFINITION 2.3. We shall say that a function $a$ is admissible, for short $a \in$ $A F[0,1]$, iff $a:[0,1] \rightarrow \mathbb{R}, \partial_{x} a \in \mathrm{J}-\mathrm{R}$ and $a(0)=a_{b}, a(1)=a_{e}$, where $a_{b}, a_{e}$ are given numbers. Here, $\partial_{x} a$ denotes the set-valued Clarke differential of $a$. 
In [22, Definition 2.2], a similar notion of 'faceted functions' appeared. The main difference is that here we admit degenerate facets, while there they were explicitly excluded from considerations.

We note that the above definition restricts the behavior of admissible function at the boundary of the domain. Namely, if $a \in A F$, then $a$ is monotone on an interval $\left[0, x_{0}\right)$ for some $x_{0} \in(0,1)$ and either

$$
a\left(x_{0}\right)>a(0) \text { or } a\left(x_{0}\right)<a(0) .
$$

By the same token, $a$ is monotone on an interval $\left(x_{0}, 1\right]$ for some $x_{0} \in(0,1)$ and either

$$
a\left(x_{0}\right)>a(1) \text { or } a\left(x_{0}\right)<a(1) .
$$

Thus, the Dirichlet boundary condition makes immobile any facet touching the boundary. Hence, such facets behave as if they had zero curvature.

A composition of multivalued operators requires proper preparations. Due to the needs of our paper, we restrict ourselves to a definition of

$$
\operatorname{sgn} \bar{o} \alpha
$$

for a suitable class of multivalued operators $\alpha$. It is most important to define this composition in the interior of the domain we work with. See also [30,31]. Let us also notice that $\operatorname{sgn} \bar{o} \alpha$ is a special selection of $\operatorname{sgn} \circ \alpha$. Any of those selections is called a Cahn-Hoffman vector field.

DEFINITION 2.4. Let us suppose that $\beta$ is admissible and $\partial_{x} \beta=\alpha \in \mathrm{J}-\mathrm{R}[0,1]$. The definition of $\operatorname{sgn} \bar{o} \alpha$ is pointwise. Let us first consider $x \in[0,1] \backslash \Xi_{\text {ess }}(\alpha)$. Then, there exists an interval $(a, b)$ containing $x$ and such that either $\beta$ is increasing on $(a, b)$ or decreasing. In the first case, we set

$$
\operatorname{sgn} \bar{\circ} \alpha(x)=1 \text {; }
$$

if $\beta$ is decreasing on $(a, b)$, then we set

$$
\operatorname{sgn} \bar{\circ} \alpha(x)=-1 \text {. }
$$

We note that the set $[0,1] \backslash \Xi_{\text {ess }}(\alpha)$ is a finite sum of open intervals, on each of them function $\beta$ is monotone. Furthermore, the end points of $[0,1]$ cannot belong to $\Xi_{\text {ess }}(\alpha)$.

Now, let us consider $x \in \Xi_{e s s}(\alpha)$, then there is $[p, q]$ a connected component of $\Xi_{e s s}(\alpha)$ containing $x$. If $F(p, q)$ is a convex facet of $\beta$, then we set,

$$
\operatorname{sgn} \bar{o} \alpha(x)=\frac{2}{q-p} x-\frac{2 p}{q-p}-1 \text { for } x \in[p, q] \text {. }
$$

If $F(p, q)$ is a concave facet of $\alpha$, then we set,

$$
\operatorname{sgn} \bar{\alpha} \alpha(x)=-\frac{2}{q-p} x+\frac{2 p}{q-p}+1 \text { for } x \in[p, q] \text {. }
$$


Now, we are in a position to state main results being also a justification of the notion of almost classical solutions to our system.

THEOREM 2.1. Let $u_{0} \in A F[0,1], L\left(u_{0, x}\right)>0$ with $u_{0}(0)=a_{b}$ and $u_{0}(1)=a_{e}$, then the system (1.1) admits unique solution in the sense specified by (3.15) and such that

$$
u_{x} \in L_{\infty}(0, T ; \mathrm{J}-\mathrm{R}[0,1]) .
$$

Moreover, $u$ is an almost classical solution, that is, it fulfills (1.1) in the following sense

$$
\begin{aligned}
& u_{t}-\partial_{x} \operatorname{sgn} \bar{\circ} u_{x}=0 \quad \text { in }[0,1] \times(0, T) \text {, } \\
& u(0, t)=a_{b}, \quad u(1, t)=a_{e} \text { for } t \in[0, T), \\
& \left.u\right|_{t=0}=u_{0} \quad \text { on }[0,1] \text {, }
\end{aligned}
$$

where the time derivative in (2.12) exists for all time instances, except for at most a finite number of exceptions, the $x$ derivative exists for at most a finite number of exceptions. Additionally, $u(\cdot, t) \in A F[0,1]$ for $t \in[0, T]$.

We study a second order parabolic equation with the goal of establishing existence of almost classical solutions. This is why we do not consider general data in $L_{2}$, but those which are more natural for this problem, where the jumps in $u_{x}$ and their number matter most. This is why we look for $u$, which not only belongs to $B V$, that is, $u(\cdot, t) \in B V$, but also $u(\cdot, t) \in A F$. In addition, the necessity of introducing essential facets will be explained.

An improvement of the above result, showing a regularization effects, is the following.

THEOREM 2.2. Let $u_{0}$ be as in Theorem above, but $L\left(u_{0, x}\right)=0$. Then, there exists a unique mild solution to (1.1), which is almost classical and it fulfills (2.12). Furthermore, $L\left(u_{x}(t)\right)>0$ for $t>0$.

The second theorem shows that the class of functions with nondegenerate facets is typical, and each initially degenerate essential facet momentarily evolves into an nontrivial interval. Furthermore, creation of such a singularity is impossible. In order to explain this phenomena, let us analyze the following very important example related to analysis of nonlinear elliptic operator defined by subdifferential of (1.3).

We first recall the basic definition. We say that $w \in \partial \mathcal{J}(u)$ iff $w \in L_{2}(a, b)$ and for all $h \in L_{2}(a, b)$ the inequality holds,

$$
\mathcal{J}(u+h)-\mathcal{J}(u) \geq(w, h)_{2} .
$$

Here $(f, g)_{2}$ stands for the regular inner product in $L_{2}(a, b)$. We also say that $v \in$ $D(\partial \mathcal{J})$, that is, $v$ belongs to the domain of $\partial \mathcal{J}$ iff $\partial \mathcal{J}(v) \neq \emptyset$.

We state here our fundamental example. We recall (1.3) and for the sake of convenience, for a while, we set $(a, b)=(-1,1)$. Then, we make the following observation. 
LEMMA 2.1. Function $\frac{1}{2} x^{2}$ does not belong to $D(\partial \mathcal{J})$.

Proof. If $\frac{1}{2} x^{2} \in D(\partial \mathcal{J})$, then there existed $w \in L_{2}(-1,1)$ such that for all $\phi \in$ $C_{0}^{\infty}(-1,1)$ and $t \in \mathbb{R}$

$$
\int_{(-1,1)}\left(\left|x+t \phi_{x}\right|-|x|\right) \mathrm{d} x \geq t \int_{(-1,1)} w \phi \mathrm{d} x .
$$

We restrict ourselves to $\phi$ such that $\phi \in C_{0}^{\infty}(-\delta, \delta)$ and supp $\phi_{x}[-\delta,-\delta / 2] \cup[\delta / 2, \delta]$. Additionally, $\phi_{x}(t)>0$ for $t \in(-\delta,-\delta / 2), \phi_{x}(t)<0$ for $t \in(\delta / 2,-\delta)$ and $\phi(-\delta)=\phi(\delta)=0, \phi(t)=1$ for $t \in(-\delta / 2, \delta / 2)$. Next, let us observe that $\left|x+t \phi_{x}(x)\right|-|x|=t \phi_{x}(x) \operatorname{sgn} x$ for $\left|t \phi_{x}(x)\right| \leq \delta / 2$; we keep in mind that $\phi_{x}(t)=0$ for $t \in(-\delta / 2, \delta / 2)$.

Thus, for such $\phi$ and $t$ the r.h.s. of (2.14) equals

$$
\begin{aligned}
\int_{(-\delta / 2, \delta / 2)}\left(\left|x+t \phi_{x}(x)\right|-|x|\right) \mathrm{d} x= & \int_{(-\delta,-\delta / 2)} t \phi_{x} \cdot(-1) \mathrm{d} x \\
& +\int_{(\delta / 2, \delta)} t \phi_{x} \cdot(1) \mathrm{d} x=-2 t .
\end{aligned}
$$

Hence, we get $-2 t \geq t \int_{(-\delta, \delta)} w \phi \mathrm{d} x$, what implies for $t>0$

$$
2 \leq-\int_{(-\delta, \delta)} w \phi \mathrm{d} x \leq \int_{(-\delta, \delta)}|w| \mathrm{d} x \rightarrow 0,
$$

since $w \in L_{2}(-1,1)$. Thus, we have reached a contradiction. Hence, $\frac{1}{2} x^{2}$ cannot belong to $D(\partial \mathcal{J})$.

A description of the domain of the subdifferential $\partial \mathcal{J}$ of (1.3) is presented below in Proposition 2.1, as well as the characterization of the lower semicontinuous envelope of $\mathcal{J}$. The analysis is based on the Legendre-Fenchel transform. The authors of [20,25] studied important aspects of this issue, but they did not solve it completely. On the other hand, the analysis similar to ours is in [13] and [14]. There is a description of $D(\mathcal{J})$ for the multidimensional version of the problem we consider, see for example, [6]. It is based on Anzellotti's formula for integration by parts [8].

PROPOSITION 2.1. (a) The lower semicontinuous envelope of $\mathcal{J}$ is given by $\mathcal{J}^{* *}$, where

$$
\mathcal{J}^{* *}(u)= \begin{cases}\int_{a}^{b}|D u|+\left|a_{b}-u(a)\right|+\left|a_{e}-u(b)\right| & \text { if } u \in B V[a, b], \\ +\infty & \text { otherwise }\end{cases}
$$

(b) If $u \in D(\mathcal{J})$, then

$$
\begin{array}{r}
\partial \mathcal{J}(u)=\left\{w=-\phi^{\prime}: \phi \in H^{1}((a, b) ;[-1,1]), D^{+} u(\{\phi<1\})=0\right. \\
=D^{-} u(\{\phi>-1\}) .
\end{array}
$$

Here, $D^{+} u$ (resp. $D^{-} u$ ) denotes the positive (resp. negative) part of measure $D u$. 
Proof. We begin by recalling the Legendre-Fenchel transform. If $\mathcal{J}: L^{2}(a, b) \rightarrow$ $(-\infty,+\infty]$ is a proper functional, then for $w \in L^{2}(a, b)$ we set (see [35])

$$
\mathcal{J}^{*}(w)=\sup _{u \in L^{2}(a, b)}(w, u)_{2}-\mathcal{J}(u)
$$

It is a well-know fact that $\mathcal{J}^{*}$ is convex and lower semicontinuous, in addition $\mathcal{J}^{* *}$ is the lower semicontinuous envelope of $\mathcal{J}$. Thus, we shall calculate $\mathcal{J}^{*}$ and $\mathcal{J}^{* *}$. Let us suppose that $w \in L^{2}(a, b)$, then there is $\phi \in H^{1}(a, b)$ such that $w=-\phi^{\prime}$. We also notice that if $u \in B V[a, b]$, then for such $\phi$, we have the following integration by parts formula,

$$
\int_{a}^{b} \phi D u+\int_{a}^{b} \phi^{\prime} u \mathrm{~d} x=\int_{a}^{b} D(u \phi)=(u \phi)(b)-(u \phi)(a) .
$$

In order to calculate $\mathcal{J}^{*}(w)$, it is sufficient to consider only $u \in D(\mathcal{J})$ in the formula below,

$$
\begin{aligned}
\int_{a}^{b} w u \mathrm{~d} x-\int_{a}^{b}|D u| & =-\int_{a}^{b} \phi^{\prime} u \mathrm{~d} x-\int_{a}^{b}|D u| \\
& =\int_{a}^{b} \phi D u-\int_{a}^{b}|D u|+a_{b} \phi(a)-a_{e} \phi(b) .
\end{aligned}
$$

Let us first suppose that $w$ is such that $\phi$ may be so chosen that, for all $x \in[a, b]$, we have $\phi(x) \in[-1,1]$. We claim that (2.20) implies that

$$
\mathcal{J}^{*}(w)=a_{b} \phi(a)-a_{e} \phi(b)
$$

Indeed, for this choice of $\phi$, we notice that

$$
\int_{a}^{b} \phi D^{+} u-\int_{a}^{b} D^{+} u-\int_{a}^{b} \phi D^{-} u-\int_{a}^{b} D^{-} u \leq 0 .
$$

Moreover, the equality holds for any $u \in D(\mathcal{J})$ such that $D^{-} u(\{\phi>-1\})=$ $0, D^{+} u(\{\phi<1\})=0$. Hence, (2.21) holds. If on the other hand $\phi \geq-1$ and the set $\{\phi(x)>1\}$ has positive measure (or $\phi \leq 1$ and the set $\{\phi(x)<-1\}$ has positive measure), then it is easy to deduce that $\mathcal{J}^{*}(w)=+\infty$.

Since we can choose $\phi$ up to a constant, we infer that

$$
\mathcal{J}^{*}(w)= \begin{cases}a_{b} \phi(a)-a_{e} \phi(b) & \text { if } w=-\phi^{\prime}, \phi \in H^{1}((a, b) ;[-1,1]), \\ +\infty & \text { otherwise. }\end{cases}
$$

Let us calculate $\mathcal{J}^{* *}$; for this purpose, we take any $u \in L^{2}(a, b)$ and $w \in D\left(\mathcal{J}^{*}\right)$ and we consider

$$
(u, w)_{2}-\mathcal{J}^{*}(w)=-\int_{a}^{b} u \phi^{\prime} \mathrm{d} x-\left(a_{b} \phi(a)-a_{e} \phi(b)\right) .
$$


Taking supremum with respect to $w$ implies that $\mathcal{J}^{* *}(u)$ is finite if and only if $u \in$ $B V[a, b]$. Hence, integration by parts in (2.23) yields,

$$
(u, w)_{2}-\mathcal{J}^{*}(w)=\int_{a}^{b} \phi D u-(u(b) \phi(b)-u(a) \phi(a))-\left(a_{b} \phi(a)-\phi(b)\right) .
$$

It is now easy to see that $\mathcal{J}^{* *}$ is given by formula (2.17). We notice that $\mathcal{J}^{* *}$ and $\mathcal{J}$ coincide on the domain of $\mathcal{J}$. Hence, (a) follows.

In order to establish (b), we use a well-know equivalence, see [35],

$$
w \in \partial \mathcal{J}^{* *}(u) \Leftrightarrow u \in \partial \mathcal{J}^{*}(w) \Leftrightarrow \int_{a}^{b} w u \mathrm{~d} x=\mathcal{J}^{* *}(u)+\mathcal{J}^{*}(w) .
$$

As a result, we conclude that for $u \in D(\mathcal{J})$ formula (2.18) is valid. Our claim follows.

At the end, we mention a result describing the asymptotics of solutions, proved in the last section.

THEOREM 2.3. There is finite $t_{\text {ext }}>0$ such that the solution $u$ reaches a steady state at $t_{\text {ext }}$, that is, $u(t)=u\left(t_{\text {ext }}\right)$ for $t>t_{\text {ext }}$. Moreover, we have an explicit estimate for $t_{\text {ext }}$ in terms of $u_{0}$, see (5.1).

Analysis for piecewise constant data in [25] also lead to observation of the finite extinction time, but without estimating it.

The above result shows that the limit of any solution, as time goes to infinity, is always a monotone function, and this will be proved and illustrated in Sect. 5. Similar results has been proved in [25], but with no estimate of the stopping time. There we present numerical simulations based on the analysis of system (1.1). It is interesting to note that in comparison with [19] who deals with the multidimensional case, our computations do not contain any discretization error. A rich possibility of stationary states is allowed thanks to Dirichlet boundary conditions, see also [27].

Note that such picture is impossible for Neumann boundary constraints, for which there are only trivial/constant equilibria.

\section{Yosida approximation}

The central object for our considerations is the Yosida approximation to $-\partial_{x} \operatorname{sgn} \partial_{x}$. First, we introduce an auxiliary notion of a nonlinear resolvent operator to the following problem,

$$
\lambda u-\frac{d}{\mathrm{~d} x} \operatorname{sgn}\left(u_{x}\right)=\lambda v \quad \text { on }[0,1], \quad u=v \quad \text { at } \partial[0,1]
$$

where $v$ is a given element of $L_{2}(0,1)$. 
DEFINITION 3.1. An operator assigning to $v \in \mathrm{J}-\mathrm{R}$ a unique solution, $u \in \mathrm{J}-\mathrm{R}$, to (3.1) will be called the resolvent of $A=-\partial_{x} \operatorname{sgn} \partial_{x}$ and we denote it by $u=$ $R(\lambda, A) v$.

Now, we may introduce the Yosida approximation to $A$.

DEFINITION 3.2. Let us assume that $A=-\partial_{x} \operatorname{sgn} \partial_{x}$ is as above and $\lambda>0$. An operator $A_{\lambda}: \mathrm{J}-\mathrm{R} \rightarrow \mathrm{J}-\mathrm{R}$ given by $A_{\lambda} u=\lambda(u-R(\lambda, A)(\lambda u))$ is called the Yosida approximation of $A$.

Since the notion of Yosida approximation seems well-understood, we will use it to explain the meaning of $A$. For this purpose, we will fix $w \in \mathrm{J}-\mathrm{R}$ and $\lambda>0$. We set $u^{\lambda}:=R(\lambda, A) w$. We will look more closely at $A_{\lambda}\left(u^{\lambda}\right)$.

THEOREM 3.1. Let us assume that $w \in A F[0,1]$, that is, $w_{x} \in \mathrm{J}-\mathrm{R}$, then there exists a unique solution to

$$
\lambda u+A(u)=\lambda w \quad \text { in }(0,1), \quad u(0)=w(0), u(1)=w(1),
$$

denoted by $u^{\lambda}$, fulfilling

$$
\left\|u_{x}^{\lambda}\right\|_{B V[0,1]} \leq\left\|w_{x}\right\|_{B V[0,1]} .
$$

Moreover, there is $\lambda_{0}>0$ such that $K_{\text {ess }}\left(u_{x}^{\lambda}\right)=K_{\text {ess }}\left(w_{x}\right)$ for $\lambda>\lambda_{0}$ with $\left\|u_{x}^{\lambda}\right\|_{\mathrm{J}-\mathrm{R}} \leq$ $\left\|w_{x}\right\|_{\mathrm{J}-\mathrm{R}}$. Furthermore, if $L\left(w_{x}\right)=d>0$, equation (3.2) can be restated as follows

$$
\lambda u^{\lambda}-\partial_{x} \operatorname{sgn} \bar{o} u_{x}^{\lambda}=\lambda w+V(\lambda, x),
$$

where $V(\lambda, x) \rightarrow 0$ in $L_{q}$ for all $q<\infty$ as $\lambda \rightarrow \infty$. In addition

$$
A_{\lambda}\left(u^{\lambda}\right) \rightarrow-\partial_{x} \operatorname{sgn} \bar{o} w_{x} \text { in } L_{q}(0,1) \text { with } q<\infty .
$$

Proof. We would like to present an independent proof of existence of solutions to system (3.2). For this purpose, we restrict ourselves to $w \in A F$ and for sufficiently large $\lambda$. A simple construction of $u^{\lambda}$ for a given $w$ based upon Lemma 3.1 is presented below.

Our assumptions give us

$$
\Xi_{e s s}\left(w_{x}\right)=\bigcup_{i=1}^{K_{e s s}\left(w_{x}\right)}\left[a_{*}^{i}, b_{*}^{i}\right]
$$

with $a_{*}^{i} \leq b_{*}^{i}$. Moreover, $a_{*}^{1}>0$ and $b_{*}^{K_{\text {ess }}\left(w_{x}\right)}<1$.

Below, we present a construction of $u^{\lambda}$. Namely, we consider system (3.2) in a neighborhood of preimage of an essential facet $\left[a_{*}^{i}, b_{*}^{i}\right]$ of $w$ (it may be degenerate), and we prescribe the evolution of this facet. If $\lambda$ is sufficiently large, then we keep the number $K_{\text {ess }}$ constant. 
LEMMA 3.1. Let us suppose that $w$ satisfies the assumptions of Theorem 3.1. Then, for sufficiently large $\lambda$, and for each $i=1, \ldots, K_{\text {ess }}\left(w_{x}\right)$, there exist monotone functions $\lambda \mapsto a_{i}(\lambda)$ and $\lambda \mapsto b_{i}(\lambda)$, which are solutions to the following problem,

$$
\left(b^{i}(\lambda)-a^{i}(\lambda)\right) w\left(a^{i}(\lambda)\right)=\int_{a^{i}(\lambda)}^{b^{i}(\lambda)} w+2 \frac{1}{\lambda} \operatorname{sgn} \kappa_{\left[a_{*}^{i}, b_{*}^{i}\right]}, \quad w\left(b^{i}(\lambda)\right)=w\left(a^{i}(\lambda)\right) .
$$

These solutions are defined locally, that is, in a neighborhood of $\left[a_{*}^{i}, b_{*}^{i}\right]$.

We recall that, the transition numbers $\operatorname{sgn} \kappa_{\left[a_{*}^{i}, b_{*}^{i}\right]}$ were defined in (2.2), (2.3). Additionally, we require

$$
\begin{array}{r}
a^{1}(\lambda)>0, \quad b^{K_{\text {ess }}\left(w_{x}\right)}(\lambda)<1 \text { and } b^{i}(\lambda)<a^{i+1}(\lambda) \\
\text { for } i=1, \ldots, K_{\text {ess }}\left(w_{x}\right)-1 .
\end{array}
$$

However, if $\lambda_{0}$ is the greatest lower bound of $\lambda$ as above, then one of the three possibilities occurs,

$$
a^{1}\left(\lambda_{0}\right)=0 \text { or } b^{K\left(w_{x}\right)}\left(\lambda_{0}\right)=1 \text { or } a^{i}\left(\lambda_{0}\right)=b^{i+1}\left(\lambda_{0}\right) .
$$

It is worthwhile to underline that the lemma holds if $L\left(w_{x}\right)=0$, too.

Proof. Fix $i$ in $\left\{1, \ldots, K_{e s s}\left(w_{x}\right)\right\}$. Problem (3.6) comes from integration of (3.2) over a neighborhood of facet $\left[a_{*}^{i}, b_{*}^{i}\right]$. For $\tau \in \mathbb{R}$ in a neighborhood of zero, such that $\tau \operatorname{sgn} \kappa_{\left[a_{*}^{i}, b_{*}^{i}\right]}>0$, we set

$$
\begin{aligned}
& \bar{a}^{i}(\tau)=\min \left(\left.w\right|_{\left[b_{*}^{i-1}, a_{*}^{i}\right]}\right)^{-1}\left(w\left(a_{*}^{i}\right)+\tau\right), \\
& \bar{b}^{i}(\tau)=\max \left(\left.w\right|_{\left[b_{*}^{i}, a_{*}^{i+1}\right]}\right)^{-1}\left(w\left(b_{*}^{i}\right)+\tau\right) .
\end{aligned}
$$

These quantities are well defined, because functions $\left.w\right|_{\left[b_{*}^{i-1}, a_{*}^{i}\right]}$ and $\left.w\right|_{\left[b_{*}^{i}, a_{*}^{i+1}\right]}$ are monotone. If these functions are strictly monotone, then $w^{-1}\left(w\left(b_{*}^{i}\right)+\tau\right)$ is strictly monotone too, so the $\min / \max$ are redundant. However, if there exists $\{\alpha\} \neq[\alpha, \beta] \subset$ $\Xi(w)$ and $[\alpha, \beta] \subset\left[b_{*}^{i-1}, a_{*}^{i}\right]$ (resp. $[\alpha, \beta] \subset\left[b_{*}^{i}, a_{*}^{i+1}\right]$, then $\left(\left.w\right|_{\left[b_{*}^{i-1}, a_{*}^{i}\right]}\right)^{-1}$ (resp. $\left.\left(\left.w\right|_{\left[b_{*}^{i}, a_{*}^{i+1}\right]}\right)^{-1}\right)$ is a maximal monotone graph and min/max makes $\bar{a}^{i}(\cdot)\left(\operatorname{resp} . \bar{b}^{i}(\cdot)\right)$ single valued and discontinuous. However, the function

$\tau \mapsto\left(\bar{b}^{i}(\tau)-\bar{a}^{i}(\tau)\right) w\left(\bar{a}^{i}(\tau)\right)-\int_{\bar{a}^{i}(\tau)}^{\bar{b}^{i}(\tau)} w(s) \mathrm{d} s=: F_{i}(\tau), \quad i=1, \ldots, K_{\text {ess }}\left(w_{x}\right)$,

is continuous. Indeed, if $\tau_{0}$ is a point, where $\bar{a}^{i}$ and $\bar{b}^{i}$ are continuous, then this statement is clear. Let us suppose that at $\tau_{0}$ function $\bar{a}^{i}$ has a jump (the argument for $\bar{b}^{i}$ is the same). Then, $\left[\bar{a}^{i}\left(\tau_{0}\right), \beta\right] \subset \Xi\left(w_{x}\right)$, where $\bar{a}^{i}\left(\tau_{0}\right)<\beta$ and for any $x \in\left[\bar{a}^{i}\left(\tau_{0}\right), \beta\right]$ we have

$$
\begin{array}{r}
\left(\bar{b}^{i}\left(\tau_{0}\right)-\bar{a}^{i}\left(\tau_{0}\right)\right) w\left(\bar{a}^{i}\left(\tau_{0}\right)\right)-\int_{\bar{a}^{i}\left(\tau_{0}\right)}^{\bar{b}^{i}\left(\tau_{0}\right)} w(s) \mathrm{d} s \\
=\left(\bar{b}^{i}\left(\tau_{0}\right)-x\right) w(x)-\int_{x}^{\bar{b}^{i}(\tau)} w(s) \mathrm{d} s .
\end{array}
$$


This is so, because we notice that $w$ restricted to $\left[\bar{a}^{i}\left(\tau_{0}\right), \beta\right]$ is constant and equal to $w\left(a_{*}^{i}\right)+\tau_{0}$. Moreover,

$$
\begin{aligned}
\int_{\bar{a}^{i}\left(\tau_{0}\right)}^{\bar{b}^{i}\left(\tau_{0}\right)} w(s) \mathrm{d} s & =\int_{\bar{a}^{i}\left(\tau_{0}\right)}^{x} w(s) \mathrm{d} s+\int_{x}^{\bar{b}^{i}\left(\tau_{0}\right)} w(s) \mathrm{d} s \\
& =\left(x-\bar{a}^{i}\left(\tau_{0}\right)\right)\left(w\left(a_{*}^{i}\right)+\tau_{0}\right)+\int_{x}^{\bar{b}^{i}\left(\tau_{0}\right)} w(s) \mathrm{d} s .
\end{aligned}
$$

Hence, our claim follows, that is, continuity of $F_{i}, i=1, \ldots, K_{e s s}(w)$. Indeed, let us suppose that $\tau_{n}$ converges from one side to $\tau_{0}$ (the side, left or right, depends upon $\operatorname{sgn} \kappa_{\left[a_{*}^{i}, b_{*}^{i}\right]}$ ) so that $\lim _{n \rightarrow \infty} \bar{a}^{i}\left(\tau_{n}\right)=\gamma$, where $\gamma=\bar{a}^{i}\left(\tau_{0}\right)$ or $\gamma=\beta$. Then, due to (3.10) we deduce continuity of $F_{i}$.

If we take $\lambda$ sufficiently large, then $\frac{2}{\lambda} \operatorname{sgn} \kappa_{\left[a_{*}^{i}, b_{*}^{i}\right]}$ is in the range of $F_{i}$, that is, there exists $\tau_{i}=\tau_{i}(\lambda)$ such that $F_{i}(\tau(\lambda))=\frac{2}{\lambda} \operatorname{sgn} \kappa_{\left[a_{*}^{i}, b_{*}^{i}\right]}$. If we further make $\lambda$ larger, then we can make sure that for each $i=1, \ldots, K_{\text {ess }}\left(w_{x}\right)$ we have $\bar{b}^{i-1}\left(\tau_{i}(\lambda)\right)<\bar{a}^{i}\left(\tau_{i}(\lambda)\right)$ and $\bar{b}^{i}\left(\tau_{i}(\lambda)\right)<\bar{a}^{i+1}\left(\tau_{i}(\lambda)\right)$. Thus, we set $a^{i}(\lambda):=\bar{a}^{i}\left(\tau_{i}(\lambda)\right), b^{i}(\lambda):=\bar{b}^{i}\left(\tau_{i}(\lambda)\right)$. Let us define $\lambda_{0}$ to be the inf of $\lambda$ 's constructed above.

We see that for $\lambda_{0}$ one of the inequalities $a^{1}\left(\lambda_{0}\right)>0, b^{i}\left(\lambda_{0}\right)<a^{i+1}\left(\lambda_{0}\right), i=$ $1, \ldots, K_{\text {ess }}\left(w_{x}\right)-1, b^{K_{\text {ess }}\left(w_{x}\right)}\left(\lambda_{0}\right)<1$ becomes equality.

This lemma permits us to define the function $u$ for $\lambda \geq \lambda_{0}$,

$$
u^{\lambda}= \begin{cases}w & \text { for } x \in[0,1] \backslash \bigcup_{i=1}^{K_{e s s}\left(w_{x}\right)}\left[a^{i}(\lambda), b^{i}(\lambda)\right], \\ w\left(a^{i}\right) & \text { for } x \in\left[a^{i}(\lambda), b^{i}(\lambda)\right] .\end{cases}
$$

We notice that $K_{\text {ess }}\left(u_{x}^{\lambda}\right)=K_{\text {ess }}\left(w_{x}\right)$ and $\Xi_{\text {ess }}\left(u_{x}^{\lambda}\right)=\bigcup_{i=1}^{K_{e s s}\left(u_{x}^{\lambda}\right)}\left[a^{i}, b^{i}\right]$, provided that $\lambda>\lambda_{0}$.

Let us analyze what happens at $\lambda=\lambda_{0}$. We know that one of the three possibilities in (3.8) occurs. We notice that if $a^{1}\left(\lambda_{0}\right)=0$ or $b^{K_{\text {ess }}\left(w_{x}\right)}\left(\lambda_{0}\right)=1$, then a facet of $u^{\lambda}$ touches the boundary. Subsequently this facet becomes a zero curvature facet, for it is immobile. This is a simple consequence of Dirichlet boundary conditions which do not admit any evolution of facets touching the boundary.

Let us look at the case $b^{i}\left(\lambda_{0}\right)=a^{i+1}\left(\lambda_{0}\right)$ for an index $i$. Thus, we obtain the phenomenon of facet merging. In both cases the structure of the set $\Xi_{\text {ess }}\left(u_{x}^{\lambda}\right)$ will be different from $\Xi_{e s s}\left(w_{x}\right)$. As a result, we have

$$
K_{\text {ess }}\left(u_{x}^{\lambda}\right)<K_{\text {ess }}\left(w_{x}\right) .
$$

It is worth stressing that at the moment $\lambda=\lambda_{0}$ more than two facets may merge, so we cannot control the decrease of number $K$. In this case we slightly modify (3.11), since the structure of $\Xi_{\text {ess }}\left(u_{x}^{\lambda}\right)$ is different from $\Xi_{e s s}\left(w_{x}\right)$. It is sufficient to notice that the number of elements in the decomposition (3.5) has decreased.

It is clear that for $\lambda \geq \lambda_{0}$, we have $K_{e s s}\left(u_{x}^{\lambda}\right) \leq K_{\text {ess }}\left(w_{x}\right)$ and by the construction, (3.11) it is also obvious that (see Definition 2.1)

$$
\left\|D u_{x}^{\lambda}\right\| \leq\left\|D w_{x}\right\|
$$


Note that the boundary conditions are given, so (3.13) controls the whole norm of $u^{\lambda}$.

Once we constructed a solution $u^{\lambda}$ by (3.11), we shall discuss the question: in what sense does it satisfy equation (1.2). One hint is given in the process of construction $a^{i}(\lambda)$ and $b^{i}(\lambda)$. This is closely related to ideas in [29]. If we stick with differential inclusions, then formula

$$
u-w-\frac{1}{\lambda} \frac{d}{\mathrm{~d} x} \operatorname{sgn} u_{x} \ni 0
$$

leads to difficulties, because we did not provide any definition of the last term on the left-hand-side (1.h.s. for short).

Here comes our meaning of a mild solution: for each $x \in[0,1]$, the following inclusion must hold

$$
\int_{0}^{x}(u-w) \mathrm{d} x^{\prime}-\left.\frac{1}{\lambda} \operatorname{sgn} u_{x}\right|_{0} ^{x} \ni 0 .
$$

We shall keep in mind that at $x=0$, we have $u=w$ (for the sake of simplicity of notation we shall suppress the superscript $\lambda$, when this does not lead into confusion).

In order to show that $u$ fulfills (3.15), we will examine a neighborhood of the first component of $\Xi_{\text {ess }}\left(u_{x}\right)$, that is, $\left[a^{1}, b^{1}\right]$. We take $x \in\left[0, a^{1}\right)$, then $u=w$ on $[0, x]$. Thus, it is enough to check whether $\frac{1}{\lambda}\left(\operatorname{sgn} u_{x}(0)-\operatorname{sgn} u_{x}(x)\right) \ni 0$. We notice that on $[0, x] \subset\left[0, a_{1}\right)$ function $u$ is monotone. As a result, $\operatorname{sgn} u_{x}(0)$ and $\operatorname{sgn} u_{x}(x)$ may equal 1 or $[-1,1]$, provided that $u$ is increasing. If on the other hand, $u$ is decreasing on $[0, x]$, then $\operatorname{sgn} u_{x}(0)$ and $\operatorname{sgn} u_{x}(x)$ are equal to -1 or $[-1,1]$. If any of these possibilities occurs, then (3.15) is fulfilled.

We shall continue after assuming for the sake of definiteness that facet $F\left(a^{1}, b^{1}\right)$ is convex. The argument for a concave facet is analogous.

Let us consider $x \in\left[a^{1}, b^{1}\right]$. We interpret $\operatorname{sgn} t$ as a multivalued function such that $\operatorname{sgn} 0=[-1,1]$. Then, we have for $x \in\left[a^{1}, b^{1}\right]$

$$
\int_{0}^{x}(u-w) \mathrm{d} x^{\prime}-\frac{1}{\lambda}[-1,1]+\left.\frac{1}{\lambda} \operatorname{sgn} u_{x}\right|_{x^{\prime}=0} \ni 0 .
$$

Since we assumed that the facet $F\left(a^{1}, b^{1}\right)$ is convex, from (3.6) we find that $0 \leq$ $\int_{0}^{x}(u-w) \mathrm{d} x^{\prime} \leq \frac{2}{\lambda}$. By the assumption, we know that $\left.\operatorname{sgn} u_{x}\right|_{x^{\prime}=0} \ni-1$. Hence,

$$
\int_{0}^{x}(u-w) \mathrm{d} x^{\prime}-\frac{1}{\lambda} \in \frac{1}{\lambda}[-1,1] .
$$

This shows (3.15) again. In case $F\left(a^{1}, b^{1}\right)$ is concave, the argument is analogous. Let us now consider $x \in\left(b^{1}, a^{2}\right]$, then we have

$$
\begin{aligned}
& \int_{0}^{x}(u-w) \mathrm{d} x^{\prime}-\left.\frac{1}{\lambda} \operatorname{sgn} u_{x}\right|_{0} ^{x}=\int_{0}^{a^{1}}(u-w) \mathrm{d} x^{\prime}-\left.\frac{1}{\lambda} \operatorname{sgn} u_{x}\right|_{0} ^{a^{1}}+\int_{a^{1}}^{b^{1}}(u-w) \mathrm{d} x^{\prime} \\
& \quad-\left.\frac{1}{\lambda} \operatorname{sgn} u_{x}\right|_{a^{1}} ^{b^{1}}+\int_{b^{1}}^{x}(u-w) \mathrm{d} x^{\prime}-\left.\frac{1}{\lambda} \operatorname{sgn} u_{x}\right|_{b^{1}} ^{x} \\
& =I_{1}+I_{2}+I_{3} .
\end{aligned}
$$


Here, we do have the freedom of choosing $\operatorname{sgn} u_{x}$ at $x=b^{1}$. Namely, we set $\operatorname{sgn} u_{x}\left(b^{1}\right)=-1$. We also know that $\operatorname{sgn} u_{x}\left(a^{1}\right)=1$.

We recall that by the very construction of $a^{1}$ and $b^{1}$, we have $I_{2}=0$. Subsequently, we notice that the argument performed for $x \in\left[0, a^{1}\right)$ applies also to $x \in\left(b^{1}, a^{2}\right]$, Thus,

$$
\begin{aligned}
I_{1}+I_{2}+I_{3} & =-\frac{1}{\lambda}\left(1-\operatorname{sgn} u_{x}(0)\right)+0-\frac{1}{\lambda}\left(-1+\operatorname{sgn} u_{x}(x)\right) \\
& =\frac{1}{\lambda}\left(-\operatorname{sgn} u_{x}(0)+\operatorname{sgn} u_{x}(x)\right) \ni 0,
\end{aligned}
$$

that is, (3.15) holds again.

Repeating the above procedure for each subsequent facet, we prove that $u$ given by (3.11) fulfills (3.15). The case $x \in\left[b^{K_{e s s}}, 1\right]$ is handled in the same way. Thus, we proved the first part of Theorem 3.1 concerning existence.

We shall look more closely at the solutions when $\lambda=\lambda_{0}$. We have then two basic possibilities: The first facet $F\left(a^{1}, b^{1}\right)$ or the last one $F\left(a^{k}, b^{k}\right)$ touches the boundary, that is, $a^{1}=0$ or resp. $b^{k}=1$. If this happens, then $F\left(0, b^{1}\right)$, resp. $F\left(a^{k}, 1\right)$, has zero curvature. Or two or more facets merge, that is, there are $i, r>0$ such that $\lim _{\lambda \rightarrow \lambda_{0}} b^{i-1}(\lambda)=b^{i-1}\left(\lambda_{0}\right)<a^{i}\left(\lambda_{0}\right)=\lim _{\lambda \rightarrow \lambda_{0}} a^{i}(\lambda)$ and $\lim _{\lambda \rightarrow \lambda_{0}} b^{i+j}(\lambda)=$ $\lim _{\lambda \rightarrow \lambda_{0}} a^{i+1+j}(\lambda), j=0,1, \ldots, r-1$, and $\lim _{\lambda \rightarrow \lambda_{0}} b^{i+r-1}(\lambda)<\lim _{\lambda \rightarrow \lambda_{0}} a^{i+r}(\lambda)$. We adopt the convention that $b^{0}=0$ and $a^{k+1}=1$.

When this happens, we have two further sub-options: an odd number of facets merge, then $F\left(a^{i}\left(\lambda_{0}\right), b^{i+r}\left(\lambda_{0}\right)\right)$ has zero curvature; or an even number of facets merge, then $\left[a^{i}\left(\lambda_{0}\right), b^{i+r}\left(\lambda_{0}\right)\right] \subset \Xi_{e s s}\left(u_{x}\right)$. Of course, it may happen that simultaneously a number of events of these types occur.

First, let us observe that $u=w$ away from the set $\left\{u_{x}=0\right\}$, so we conclude $\Xi\left(w_{x}\right) \subset \Xi\left(u_{x}\right)$. More precisely, the equality holds on a larger set. Namely, if $F\left(a^{i}, b^{i}\right)$ is a zero curvature facet and $\lambda>\lambda_{0}$, then the very construction of $a^{i}(\lambda), b^{i}(\lambda)$ implies that $u=w$ on $\left[a^{i}, b^{i}\right]$. If $u(a)=u(b)=w(a)=w(b)$, so there must be a point $c \in(a, b)$ such that $0 \in w_{x}(c)$. Thus, we obtain for any $\lambda>0$ that $K_{\text {ess }}\left(u_{x}\right) \leq K_{\text {ess }}\left(w_{x}\right)$.

Let $L\left(w_{x}\right)=d>0$, then we consider

$$
\lambda u+A_{\lambda}(u)=\lambda w \text { for } \lambda>\lambda_{0},
$$

where we suppressed the superscript $\lambda$ over $u$.

As we have already seen, taking large $\lambda$, that is, $\lambda>\lambda_{0}$, excludes the possibility of facet merging or hitting the boundary, thus $K_{\text {ess }}\left(w_{x}\right)=K_{\text {ess }}\left(u_{x}\right)$. Let us emphasize that $K_{\text {ess }}\left(u_{x}\right)$ may decrease only a finite number of times.

Let us suppose that $\left[a^{*}, b^{*}\right]$ is a connected component of $\Xi_{e s s}\left(u_{x}\right)$, that is, $a^{*}=$ $a^{i_{0}}(\lambda), b^{*}=b^{i_{0}}(\lambda)$ for an index $i_{0}$. Without loss of generality, we may assume that this facet is convex. So, integrating (3.19) over $\left[a^{*}, b^{*}\right]$, we find

$$
\int_{a^{*}}^{b^{*}} \lambda u-\int_{a^{*}}^{b^{*}} \lambda w=2 .
$$


First, we want to find an answer to the following question. What we can say about the behavior of the following quantity $\int_{a^{*}}^{a}+\int_{b}^{b^{*}}(\lambda u-\lambda w)$, where $[a, b]$ is a connected component of $\Xi_{e s s}\left(w_{x}\right)$ contained in $\left[a^{*}, b^{*}\right]$. In fact we assume, that $a=a^{i_{0}}, b=b^{i_{0}}$.

Since $d=L\left(w_{x}\right)$ is fixed and positive, we find from (3.20) that

$$
2=\int_{a^{*}}^{b^{*}} \lambda(u-w) \geq \int_{a}^{b} \lambda(u-w) \geq\left.d \lambda(u-w)\right|_{[a, b]},
$$

Because $u-w$ is monotone on $[a, b]$. As a result,

$$
\left.\lambda(u-w)\right|_{[a, b]} \leq \frac{2}{d} .
$$

Then, we conclude that $\int_{b}^{b^{*}} \lambda(u-w) \leq\left(b^{*}-b\right) \lambda\left[w\left(b^{*}\right)-w(b)\right]$. At the same time, (3.21) yields $w\left(b^{*}\right)-w(b) \leq \frac{2}{\mathrm{~d} \lambda}$. On the other hand, $w$ is monotone on set $\left(b, b^{*}\right)$. Hence, (3.21) implies that

$$
b^{*}-b \equiv b^{i_{0}}(\lambda)-b^{i_{0}} \leq W^{-1}\left(\frac{2}{\mathrm{~d} \lambda}\right)
$$

where $W^{-1}(\cdot)$ is a strictly monotone (possibly multivalued) function, equal $w^{-1}$ (restricted to an interval of monotonicity) plus a constant such that $\lim _{t \rightarrow 0^{+}} W^{-1}(t)=$ 0 . Eventually, we get

$$
\int_{b}^{b^{*}} \lambda(u-w) \leq W^{-1}\left(\frac{2}{\mathrm{~d} \lambda}\right) \frac{2}{d} \rightarrow 0 \text { as } \lambda \rightarrow \infty .
$$

Since the analysis for $\left(a^{*}, a\right)$ is the same, (3.22) and (3.23) imply that $\int_{a^{*}}^{b^{*}} \lambda(u-w)=$ $2+V(\lambda)$. Note that $V(\lambda)$ depends only on $w$, so in Sect. 4, we will study the approximation error $V(\lambda)$ and we will show uniform bounds, provided that $L\left(w_{x}\right) \geq d>0$.

Integrating (3.19) gives

$$
\int_{a^{*}}^{b^{*}} \lambda(u-w)=\int_{a^{*}}^{b^{*}}-A_{\lambda}(u)=2,
$$

but the pointwise information from the equation yields

$$
\left.\lambda(u-w)\right|_{[a, b]}=-A_{\lambda}(u)=\text { const } .
$$

Thus, taking into account (3.24) and (3.25), we get $-\left.A_{\lambda}(u)\right|_{[a, b]} \rightarrow 2 /(b-a)$ a.e. as $\lambda \rightarrow \infty$. Here, we used that $a^{*}=a^{i_{0}}(\lambda) \rightarrow a^{i_{0}}, b^{*}=b^{i_{0}}(\lambda) \rightarrow b^{i_{0}}$ as $\lambda$ goes to infinity. But $a, b$ depends only on $w$; additionally, we shall keep in mind that (3.21) via (3.19) implies that $\left|A_{\lambda}(u)\right| \leq 2 / d$ on whole $[0,1]$.

Clearly, by Definition $2.4 \partial_{x} \operatorname{sgn} \bar{o} u_{x}=\frac{2}{\left(b^{*}-a^{*}\right)}$ for $x \in\left[a^{*}, b^{*}\right]$, hence, we have proved that

$$
A_{\lambda}(u)=-\partial_{x} \operatorname{sgn} \bar{o} u_{x}+V(\lambda, x),
$$


where $V(\lambda)=\int_{a^{*}}^{b^{*}} V(\lambda, x) \mathrm{d} x$ and $V(\lambda, x) \rightarrow 0$ in at least $L_{1}(I)$. Here, we should note clearly that all depend on $\lambda$, since $a^{*}=a^{i_{0}}(\lambda), b^{*}=b^{i_{0}}(\lambda)$. We see that we have already proved that $|V(\lambda, x)| \leq \frac{2}{d}$, and $\mu(\{\operatorname{supp} V(\lambda, \cdot)\}) \rightarrow 0$ which gives a relatively strong convergence. Note that in (3.26), we are not able to obtain "pure" discontinuity in the composition $\bar{\circ}$, since we work with solutions only; hence, $\operatorname{sgn} \overline{\mathrm{o}} u_{x}^{\lambda}$ must be piecewise linear.

Next question is: whether $\partial_{x} \operatorname{sgn} \bar{o} u_{x}^{\lambda} \rightarrow \partial_{x} \operatorname{sgn} \bar{\sigma} w_{x}$ and in which space?

Let us observe that (see Definition 2.1)

$$
\left\|D u_{x}^{\lambda}\right\| \leq\left\|D w_{x}\right\| \quad \text { and } \quad u_{x}^{\lambda} \rightarrow w_{x} \text { in measure on } I .
$$

It follows that $\operatorname{sgn} \bar{o} u_{x}^{\lambda}\left|\Xi\left(w_{x}\right) \rightarrow \operatorname{sgn} \bar{o} w_{x}\right| \Xi\left(w_{x}\right)$ uniformly. We remember that $\operatorname{sgn} \bar{o} u_{x}^{\lambda}$ and sgn $\bar{o} w_{x}$ are piecewise linear functions and the set $\Xi\left(w_{x}\right)$ is independent from $\lambda$, but the case $L\left(w_{x}\right)=d>0$ implies that

$$
A\left(u^{\lambda}\right) \rightarrow-\frac{d}{\mathrm{~d} x} \operatorname{sgn} \bar{\circ} w_{x} \text { in } L_{q}(0,1) \quad q \in[1, \infty) .
$$

Theorem 3.1 is proved.

In particular, as a result of our analysis, we get that the constructed solution to (3.2) is variational.

LEMMA 3.2. Function $u^{\lambda}$ given by Theorem 3.1 is a variational solution to (3.2), that is, $u^{\lambda}$ fulfills

$$
\left(\lambda u^{\lambda}, \phi\right)+\left(\sigma(x), \phi^{\prime}\right)=(\lambda w, \phi) \quad \text { for each } \phi \in C_{0}^{\infty}(0,1)
$$

and $\sigma(x) \in \operatorname{sgn} \circ u_{x}(x)$, where here $\circ$ denotes the standard composition.

Proof. From the inclusion (3.15), we are able to find such measurable $\sigma$ that

$$
\int_{0}^{x}(u-w)-\frac{1}{\lambda} \sigma(x)+\frac{1}{\lambda} \sigma(0)=0 .
$$

Then, testing it by $\phi^{\prime}$ with $\phi \in C_{0}^{\infty}(0,1)$, we get (3.29). In particular, we already have shown that $\lambda R(\lambda, A) \lambda$ is a monotone operator in $L_{2}$.

The form of (3.14) allows to look at the system as on a semidiscretization in time with step $h=\frac{1}{\lambda}$, see (1.4) too. Our next Lemma explains that $h$ may be split into smaller steps at will. This permits to perform additional analysis at the intermediate steps.

LEMMA 3.3. Let us suppose that $v$ is absolutely continuous and $h_{1}, h_{2}>0$, the sets $\left\{v_{x}>0\right\},\left\{v_{x}<0\right\}$ are open, and they have a finite number of connected components. If $u^{1}$ is a minimizer of

$$
\mathcal{J}_{h_{1}, v}(u)=\int_{0}^{1} h_{1}\left|u_{x}\right|+\frac{1}{2}(u-v)^{2}
$$


while $u^{2}$ is a minimizer of

$$
\mathcal{J}_{h_{2}, u^{1}}(u)=\int_{0}^{1} h_{2}\left|u_{x}\right|+\frac{1}{2}\left(u-u^{1}\right)^{2}
$$

then $u^{2}$ is a minimizer of

$$
\mathcal{J}_{h, v}(u)=\int_{0}^{1} h\left|u_{x}\right|+\frac{1}{2}(u-v)^{2} \quad \text { with } h=h_{1}+h_{2} \text {. }
$$

Proof. In fact due to our assumptions, we have solutions to the equations

$$
h_{1} \frac{d}{\mathrm{~d} x} \sigma^{1}=u^{1}-v, \quad h_{2} \frac{d}{\mathrm{~d} x} \sigma^{2}=u^{2}-u^{1} .
$$

We note that the sequence of implications: $u_{x}^{2}$ is different from zero at $x$ and then $u_{x}^{1}$ has a sign there; hence, $v_{x}$ has a sign too. Moreover, if $v_{x}=0$ on an interval $(\alpha, \beta)$, then $u_{x}^{1}, u_{x}^{2}$ are zero $(\alpha, \beta)$ too.

We want to show that

$$
h \frac{d}{\mathrm{~d} x} \operatorname{sgn} u_{x}^{2}=u^{2}-v
$$

has a solution. Let us add up the two equations above. This yields,

$$
h \frac{d}{\mathrm{~d} x}\left(\frac{h_{1}}{h} \sigma^{1}+\frac{h_{2}}{h} \sigma^{2}\right)=u^{2}-v .
$$

Of course $\sigma:=\frac{h_{1}}{h} \sigma^{1}+\frac{h_{2}}{h} \sigma^{2} \in[-1,1]$. If at $x$ we have $u_{x}^{2}(x)>0$, then $v_{x}(x)>0$. Hence,

$$
\sigma(x)=\frac{h_{1}}{h} \sigma^{1}(x)+\frac{h_{2}}{h} \sigma^{2}(x)=\frac{h_{1}}{h}+\frac{h_{2}}{h}=1 .
$$

The situation is similar if $u_{x}^{2}(x)<0$. Let us suppose now that $u_{x}^{2}(x)=0$, then regardless of the sign of $u_{x}^{1}(x)$, we know that $\sigma(x) \in[-1,1]$ and, by the definition of $\sigma$, equation (3.32) is satisfied. In particular,

$$
-2 h=h \int_{\xi_{2}^{-}}^{\xi_{2}^{+}} \sigma(x) \mathrm{d} x=\int_{\xi_{2}^{-}}^{\xi_{2}^{+}}\left(v\left(\xi_{2}^{-}\right)-v(x)\right) \mathrm{d} x=\int_{\xi_{2}^{-}}^{\xi_{2}^{+}}\left(u^{2}\left(\xi_{2}^{-}\right)-v(x)\right) \mathrm{d} x .
$$

The value of this result is that it permits us to split $h$. We may say that this shows the semigroup property. This feature is effectively applied in numerical simulations in Sect. 5 . 


\section{The construction of the flow}

A key point of our construction is an approximation of the original problem based on the Yosida approximation. Here, we meet techniques characteristic for the homogeneous Boltzmann equation [18,28]. For given $\lambda, t_{0}$ and $A_{\lambda}$ defined in (1.5), we introduce the following equation for $u^{\lambda}$,

$$
\begin{aligned}
& u^{\lambda}\left(t+t_{0}\right)=u^{\lambda}\left(t_{0}\right)-\int_{t_{0}}^{t_{0}+t} A_{\lambda}\left(u^{\lambda}\right) \mathrm{d} s, \\
& u^{\lambda}\left(0, t_{0}+t\right)=a_{b}, \quad u^{\lambda}\left(1, t_{0}+t\right)=a_{e} \quad \text { for } t \in(0, T) .
\end{aligned}
$$

We stress that its solvability, established below, does not require that $L\left(u_{x}\left(t_{0}\right)\right)>0$.

LEMMA 4.1. Let us suppose that $u^{\lambda}\left(t_{0}\right) \in \mathrm{J}-\mathrm{R}(I)$, where $I=[0,1]$, then there exists a unique solution $u^{\lambda}$ to (4.1) on the time interval $\left(t_{0}, t_{0}+\frac{1}{3 \lambda}\right)$ and

$$
u^{\lambda} \in C\left(t_{0}, t_{0}+\frac{1}{3 \lambda} ; L_{2}(I)\right), \text { moreover, } \sup _{t \in\left(0, \frac{1}{3 \lambda}\right)}\left\|u^{\lambda}\left(t_{0}+t\right)\right\|_{\mathrm{J}-\mathrm{R}} \leq\left\|u^{\lambda}\left(t_{0}\right)\right\|_{\mathrm{J}-\mathrm{R}}
$$

Proof. We will first show the bounds. Let us suppose that $u^{\lambda}$ is a solution to (4.1), then Definition 3.2 and the observation $\frac{d}{\mathrm{~d} t}\left[e^{\lambda t} u^{\lambda}\right]=-e^{\lambda t} A_{\lambda}\left(u^{\lambda}\right)+\lambda e^{\lambda t} u^{\lambda}$ imply that,

$$
u^{\lambda}\left(t_{0}+t\right)=e^{-\lambda t} u^{\lambda}\left(t_{0}\right)+\int_{t_{0}}^{t_{0}+t} e^{-\lambda\left(t_{0}+t-s\right)} \lambda R(\lambda, A) \lambda u^{\lambda}(s) \mathrm{d} s .
$$

In order to obtain the estimate in $B V$, we apply Theorem 3.1, inequality (3.3), getting

$$
\begin{aligned}
\sup _{t}\left\|u_{x}^{\lambda}\right\|_{B V} & \leq e^{-\lambda t}\left\|u_{x}^{\lambda}\left(t_{0}\right)\right\|_{B V}+\sup _{t}\left\|R(\lambda, A) \lambda u^{\lambda}(t)\right\|_{B V} \int_{0}^{t} \lambda e^{-\lambda s} \mathrm{~d} s \\
& \leq e^{-\lambda t}\left\|u_{x}^{\lambda}\left(t_{0}\right)\right\|_{B V}+\sup _{t} \frac{1}{\lambda}\left\|\lambda u_{x}^{\lambda}(t)\right\|_{B V}\left(1-e^{-\lambda t}\right) .
\end{aligned}
$$

So we get

$$
\sup _{t}\left\|u_{x}^{\lambda}\right\|_{B V} \leq\left\|u_{x}^{\lambda}\left(t_{0}\right)\right\|_{B V}
$$

In order to prove existence, we fix $\lambda$ (we will omit the index $\lambda$ in the considerations below) and we define a map $\Phi: C\left(0, T ; L_{2}(I)\right) \rightarrow C\left(0, T ; L_{2}(I)\right)$ such that $v=\Phi(w)$, where

$$
v(t)=e^{-\lambda t} v_{0}+\int_{0}^{t} e^{\lambda(t-s)} \lambda R(\lambda, A) \lambda w \mathrm{~d} s .
$$

We notice that due to $\Xi\left((\lambda R(\lambda, A) \lambda w)_{x}\right) \supset \Xi\left(w_{x}\right)$, we obtain $\Xi\left(v_{0, x}\right) \subset \Xi\left(w_{x}(t)\right)$ for $t \in(0, T)$, provided that $\left.w\right|_{t=t_{0}}=v_{0}$. Combining this observation with $\left.w\right|_{t=t_{0}}=$ $v_{0}$ again yields,

$$
\Xi\left(v_{0, x}\right) \subset \Xi\left(v_{x}(t)\right) \quad \text { for } t \in(0, T) \text {. }
$$


We see that a fixed point of the above map yields a solution to (4.1) after a shift of time. For the purpose of proving existence of a fixed point of $\Phi$, we will check that $\Phi$ is a contraction. We notice that if $w, \bar{w} \in C\left(0, T ; L_{2}(I)\right)$, then monotonicity of $R(\lambda, A) \lambda$ (thanks to Lemma 3.2) implies that $\|R(\lambda, A) \lambda w-R(\lambda, A) \lambda \bar{w}\|_{L_{2}} \leq\|w-\bar{w}\|_{L_{2}}$. Hence,

$$
\begin{aligned}
& \|\Phi(w)-\Phi(\bar{w})\|_{L_{\infty}\left(0, T ; L_{2}(I)\right)} \\
& \quad \leq \int_{0}^{t} \lambda e^{-\lambda(t-s)} \mathrm{d} s\|R(\lambda, A) \lambda w-R(\lambda, A) \lambda \bar{w}\|_{L_{\infty}\left(0, T ; L_{2}(I)\right)} \\
& \quad \leq\left(1-e^{-T \lambda}\right)\|w-\bar{w}\|_{L_{\infty}\left(0, T ; L_{2}(I)\right)},
\end{aligned}
$$

that is, $\Phi$ is a contraction provided that $0<T \leq \frac{1}{3 \lambda}$. Now, Banach fixed point theorem implies immediately existence of $u^{\lambda}$, a unique solution to (4.1) in $C\left(0, T ; L_{2}(I)\right)$.

An aspect is that the solution to (4.3) can be recovered as a limit of the following iterative process $v^{k+1}=\Phi\left(v^{k}\right)$. We have to show that the fixed point belongs to a better space. For this purpose, we use estimate (4.4), which shows also that if $\left\|v_{x}^{0}\right\|_{B V}=M$, then $\left\|v_{x}^{k}\right\|_{B V} \leq M$ for all $k \in \mathbb{N}$. Moreover, convergence in $L_{2}(I)$ implies convergence in $L_{1}(I)$ and lower semicontinuity of the total variation measure (see [38, Theorem 5.2.1.]) yields $u^{\lambda} \in L_{\infty}(0, T ; B V(I))$.

Finally, we show that

$$
K_{\text {ess }}\left(u^{\lambda}\left(t_{0}+\frac{1}{3 \lambda}\right)\right) \leq K_{\text {ess }}\left(u\left(t_{0}\right)\right) .
$$

For this purpose, it is enough to prove that $u^{\lambda}\left(t_{0}+t\right)=u^{\lambda}\left(t_{0}\right.$ on $I \backslash \Xi\left(u^{\lambda}\left(t_{0}+t\right)\right)$ for all $t \leq \frac{1}{3 \lambda}$, but Theorem 3.1 implies $R(\lambda, A) \lambda u^{\lambda}=\lambda u^{\lambda}$ on $I \backslash \Xi\left(R(\lambda, A) \lambda u^{\lambda}\right)$, namely $A_{\lambda}\left(u^{\lambda}\right)=0$ at $I \backslash \Xi\left(R(\lambda, A) \lambda u^{\lambda}\right)$. Additionally (4.6) yields that $\Xi\left(u^{\lambda}\left(t_{0}\right)\right) \subset$ $\Xi\left(u^{\lambda}\left(t_{0}+\frac{1}{3 \lambda}\right)\right.$, what finishes the proof of (4.7).

Thus, the definition of the solution to (4.1) as the limit of the sequence $v^{k}$ together with (4.7) implies (4.2). The Lemma is proved.

LEMMA 4.2. Let us consider $u^{\lambda}(\cdot)$ given by Lemma 4.1. If $L\left(u^{\lambda}\left(t_{0}\right)\right)=0$, then $L\left(u^{\lambda}\left(t_{0}+\frac{1}{3 \lambda}\right)\right)>0$.

Proof. Let us assume a contrary, then there exists a degenerate facet $F\left[a^{i}, b^{i}\right]$ with $a^{i}=b^{i}$ such that all functions $u^{\lambda}\left(t_{0}+t\right)$ are convex in a neighborhood $(p, q)$ of point $a^{i}$ and they all have a minimum only in point $a^{i}$. If functions $u^{\lambda}\left(t_{0}+t\right)$ are concave, then the argument is analogous. Let us then integrate (4.1) over $\left(a^{\prime}, b^{\prime}\right)$ such that $a^{i} \in\left(a^{\prime}, b^{\prime}\right) \subset(p, q), \int_{a^{\prime}}^{b^{\prime}} u^{\lambda}\left(t_{0}+t\right)=\int_{a^{\prime}}^{b^{\prime}} u^{\lambda}\left(t_{0}\right)-\int_{t_{0}}^{t_{0}+t} \int_{a^{\prime}}^{b^{\prime}} A_{\lambda}\left(u^{\lambda}\right) \mathrm{d} s$. But $\int_{a^{\prime}}^{b^{\prime}} A_{\lambda}\left(u^{\lambda}\right)=\int_{a^{\prime}}^{b^{\prime}} \lambda\left(u^{\lambda}-R(\lambda, A) \lambda u^{\lambda}\right)=-2$, because $u^{\lambda}$ is convex on $\left(a^{\prime}, b^{\prime}\right)$. Hence, we find $\int_{a^{\prime}}^{b^{\prime}} u^{\lambda}\left(t_{0}+t\right)=\int_{a^{\prime}}^{b^{\prime}} u^{\lambda}\left(t_{0}\right)+2 t$. But, if our assumption that $a^{i}=b^{i}$ were true, then we would be allowed to pass to the limits, $a^{\prime} \rightarrow a^{i^{-}}$and $b^{\prime} \rightarrow a^{i^{-}}$ concluding that $0=0+2 t$, which is impossible for positive $t$. Thus, $u^{\lambda}\left(t_{0}+\frac{1}{3 \lambda}\right)$ does not admit degenerate facets. 
After these preparations, we finish the proofs of Theorems 2.1 and 2.2. We shall construct an approximation of solution on a fixed time interval, say [0,1]. Let us assume that $U^{\lambda}:[0,1] \times I \rightarrow \mathbb{R}$ is given as follows

$$
U^{\lambda}=u_{k}^{\lambda} \quad \text { for } t \in\left[\frac{k}{3 \lambda}, \frac{k+1}{3 \lambda}\right) \text { and } 0 \leq k<3 \lambda \text {, }
$$

where functions $\left\{u_{k}^{\lambda}\right\}$ are given by the following relations

$$
\begin{aligned}
& u_{1}^{\lambda}(t)=u_{0}-\int_{0}^{t} A_{\lambda}\left(u_{1}^{\lambda}\right) \mathrm{d} s \text { for } t \in\left(0, \frac{1}{3 \lambda}\right] \text {, } \\
& u_{k+1}^{\lambda}\left(t_{k}+t\right)=u_{k}\left(t_{k}\right)-\int_{t_{k} \ldots}^{\cdots} A_{\lambda} A_{k}\left(u_{k+1}^{\lambda}\right) \mathrm{d} s \text { for } t \in\left(0, \frac{1}{3 \lambda}\right] \text {, } \\
& u_{3 \lambda}^{\lambda}\left(t_{3 \lambda-1}+t\right)=u_{3 \lambda-1}^{\lambda}\left(t_{3 \lambda-1}\right)-\int_{t_{3 \lambda-1}}^{t_{3 \lambda-1}+t} A_{\lambda}\left(u_{3 \lambda}^{\lambda}\right) \mathrm{d} s \text { for } t \in\left(0, \frac{1}{3 \lambda}\right]
\end{aligned}
$$

and $t_{k}=\frac{k}{3 \lambda}$ for $0 \leq k<3 \lambda$. By construction we have

$$
\left\|U^{\lambda}\right\|_{L_{\infty}(0, T ; \mathrm{J}-\mathrm{R})} \leq\left\|u_{0}\right\|_{\mathrm{J}-\mathrm{R}}
$$

Now, we pass to the limit with $\lambda$. The estimates imply that $\left\|U^{\lambda}\right\|_{L_{\infty}\left(0, T ; L_{2}(I)\right)} \leq M$. Thus, we can extract a subsequence such that $U^{\lambda} \rightarrow^{*} U^{*}$ weakly*in $L_{\infty}\left(0,1 ; L_{2}(I)\right)$. Moreover, the lower semicontinuity of the total variation measure yields $\left\|U^{\lambda}(t)\right\|_{B V} \leq$ $\|u(0)\|_{B V}$ for a.e. $t \in[0,1]$. Thus, we should look closer at the limit

$$
U^{*}\left(t_{0}+t\right)=U^{*}\left(t_{0}\right)-\lim _{\lambda \rightarrow \infty} \int_{t_{0}}^{t_{0}+t} A_{\lambda}\left(U^{\lambda}\left(t_{0}+t\right)\right) \mathrm{d} s .
$$

Let us observe that for a fixed $\lambda$ the function $K_{\text {ess }}\left(U^{\lambda}(t)\right)$, taking values in $\mathbb{N}$, is decreasing, so facet merging may occur just only a finite number of times.

Let $K\left(u_{0}\right)=k^{0}$, then for a given $\lambda$, we define $T_{1}^{\lambda}$ as follows

$$
K_{\text {ess }}\left(U^{\lambda}(t)\right)=k^{0} \quad \text { for } t \in\left[0, T_{1}^{\lambda}\right) \text { and } K_{\text {ess }}\left(U^{\lambda}\left(T_{1}^{\lambda}\right)\right)<k^{0} .
$$

For a subsequence $\lim T_{1}^{\lambda}=: T_{1}$. Indeed, $T_{1}^{\lambda}=T_{1}^{\lambda^{\prime}}$ for all sufficiently large $\lambda, \lambda^{\prime}$ see Lemma 3.3, so we have here $T_{1}>0$.

In a similar manner to (4.8), we define a sequence of time instances $\left\{T_{k}\right\}_{k=1}^{m}$. By the definitions, for any $\epsilon>0$ there exists $\lambda_{\epsilon}$, such that for $\lambda>\lambda_{\epsilon}$-up to possible subsequence-we can split the time interval $[0,1]$ into following parts

$$
[0,1)=\left[0, T_{1}-\epsilon\right) \cup\left[T_{1}-\epsilon, T_{2}+\epsilon\right) \cup\left[T_{2}+\epsilon, T_{3}-\epsilon\right] \cup \cdots \cup\left[T_{m}+\epsilon, 1\right)
$$

and $K_{\text {ess }}\left(u^{\lambda}(t)\right)=K_{\text {ess }}\left(U^{*}(t)\right)$ for $t \in\left[T_{k}+\epsilon, T_{k+1}-\epsilon\right)$, so $\left\{T_{k}\right\}$ is a finite sequence of moments of time at which facets merge. In order to avoid unnecessary problems, we restrict ourselves to a suitable subsequence guaranteeing the above properties. 
Now, Theorem 3.1 yields $A_{\lambda}\left(U^{\lambda}\right) \rightarrow A\left(U^{*}\right)=-\partial_{x} \operatorname{sgn} \bar{o} U_{x}^{*}$ in $L_{q}(0,1)$ on time intervals $\left(T_{k}+\epsilon, T_{k+1}-\epsilon\right)$, since by (3.26), we control this convergence uniformly at whole intervals. So, we get

$$
U^{*}\left(t_{0}+t\right)=U^{*}\left(t_{0}\right)-\int_{t_{0}}^{t_{0}+t} A\left(U^{*}(s)\right) \mathrm{d} s,
$$

because we consider one interval $\left[T_{k}+\epsilon, T_{k+1}-\epsilon\right)$. However, crossing $T_{k}$ requires some extra care.

In order to extend the result on the whole interval $[0,1]$, it is sufficient to prolong the solution onto interval $\left[T_{k}-\epsilon, T_{k}+\epsilon\right)$. For this purpose, we can use that $u^{\lambda}$ belongs to $C\left(0,1 ; L_{1}(I)\right)$, see Lemma 4.1. Continuity of the solution allows us to cross points $T_{k}$. It follows that $\frac{d}{\mathrm{~d} t} U^{*}$ exists except points $\left\{T_{k}\right\}$ and by the properties of solutions on intervals $\left[T_{k}, T_{k+1}\right)$, we find that the right-hand-side time derivative exists everywhere, including points $\left\{T_{k}\right\}, \frac{d}{\mathrm{~d} t^{+}} U^{*}$ exists everywhere on $[0,1]$. Finally, we have shown that $U^{*}$ fulfills

$$
\frac{d}{\mathrm{~d} t^{+}} U^{*}=-\frac{d}{\mathrm{~d} x} \operatorname{sgn} \bar{\circ} U^{*}
$$

as an almost classical solution.

By construction $U^{*}(t) \in A F$, additionally Lemma 4.2 yields $L\left(U^{*}(t)\right)>0$ for $t>0$, even as $L\left(u_{0, x}\right)=0$. Moreover, the features of almost classical solutions imply that they are variational, too. Hence, the monotonicity of sgn implies immediately uniqueness to our problem. Theorems 2.1 and 2.2 are proved.

\section{Asymptotics and examples}

Here, we present the proof of Theorem 2.3, an example of an explicit solution and numerical results describing the time behavior of solutions.

\subsection{A proof of Theorem 2.3}

Here is the argument. There is a finite number $N$ of facet merging events $0=t_{0}<$ $t_{1}<\cdots<t_{N}<\infty$, when $u$ has no time derivative but only the right-time derivative. Moreover, $N \leq K_{\text {ess }}\left(u_{0, x}\right)$. We shall estimate $\max _{i=0, \ldots N-1}\left\{t_{i+1}-t_{i}\right\}$. Let us set

$B=\max \left\{a_{b}, a_{e}\right\}, b=\min \left\{a_{b}, a_{e}\right\}, \Delta_{M}=\max u_{0}(x)-B, \Delta_{m}=b-\min u_{0}(x)$,

and $\ell=1$ is the length of $I=[0,1]$. We notice that since our solution is almost classical, $u_{t}$ exists except $t \in\left\{t_{0}, t_{1}, \ldots, t_{N}\right\}$. Moreover, $u_{t}$ is the vertical velocity of $u$. It is obvious from the definition of the composition $\bar{o}$ that the absolute value of $\left(\operatorname{sgn} \bar{o} u_{x}\right)_{x}$ is bigger or equal $2 / \ell$. We notice that the distance each essential facet travels in the vertical motion between collisions is no bigger than $A=\max \left\{\Delta_{M}, \Delta_{m}, B-b\right\}$. Since we have a lower bound on the vertical velocity 
of $u$, we conclude that $\max _{i=0, \ldots N-1}\left\{t_{i+1}-t_{i}\right\} \leq A \cdot \frac{2}{\ell}$. Thus, we have the following estimate

$$
t_{\text {ext }} \leq 2 K_{\text {ess }}\left(u_{0, x}\right) A / \ell
$$

Hence, $K_{\text {ess }}\left(u_{x}\left(t_{\text {ext }}\right)\right)=0$, then thus $u(t)$ for $t \geq t_{\text {ext }}$ is a monotone function being a stationary state of the system.

\subsection{An explicit solution}

In order to illustrate the behavior of a particular solution, we take $x^{2}$ as an initial datum for (1.1). We consider this system on the interval $(-1,1)$,

$$
\begin{array}{ll}
u_{t}-\frac{d}{\mathrm{~d} x} \operatorname{sgn} u_{x}=0 & \text { in }(-1,1) \times(0, T), \\
u(-1, t)=u(1, t)=1 & \text { for } t \in(0, T), \\
\left.u\right|_{t=0}=x^{2} & \text { for } x \in(-1,1) .
\end{array}
$$

The results we proved guarantee the following form of the solution to (5.2),

$$
u(x, t)= \begin{cases}a^{2}(t) & \text { for }|x| \leq a(t), \\ x^{2} & \text { for }|x| \in(a(t), 1)\end{cases}
$$

By Definition 2.4, we get that $\left.\frac{d}{\mathrm{~d} x} \operatorname{sgn} \overline{\mathrm{o}} u_{x}\right|_{[-a(t), a(t)]}=\frac{1}{a(t)}$. Thus, by (5.2) and (5.3), we find the following equation for $a(t)$,

$$
\partial_{t} a^{2}(t)=\frac{1}{a(t)} . \text { Hence } a(t)=\sqrt[3]{\frac{3}{2}} t,
$$

in agreement with the initial datum.

Summing up the length of the facet is $2 a(t)=2 \sqrt[3]{\frac{3}{2} t}$, the speed of it is $\partial_{t} a(t) \sim t^{-2 / 3}$ and the extinction time of $u \equiv 1$ is $T_{\text {stab }}=\frac{2}{3}$. We are able to point out many such examples, and construction of them is relatively easy and known, for example, [23,24].

\subsection{Numerical simulations}

Now, we are prepared to computer implementations of our results. Simulations were done in Octave package. The main part of the program is a loop running until the graph reaches its final shape. During one step all facets (i.e., points where $0 \in \partial f$ ) are moved until (if it is possible) each of them fills the area equal to $2 h$. In the pictures shown below, we used $h=5$. The reason why it may be not possible to fill the $2 h$ area is that the moving facet may reach the boundary of the interval that it is defined on or it may reach the boundary of another facet after it filled the required area (whereas each of them moved separately may fit its domain). When any of these interactions happens, 
Table 1. Examples 1, 2, 3 (respectively) used in the simulations

\begin{tabular}{llll}
\hline Domain & $v_{1}$ & $v_{2}$ & $v_{3}$ \\
\hline$[-1.5,-1]$ & $x-2$ & $3 x^{2}+11 x$ & $3 x^{2}+11 x$ \\
{$[-1,0]$} & $-x^{2}+x+2$ & $-x^{2}+5 x+1$ & $-x^{2}+5 x+1$ \\
{$[0,1]$} & $x^{2}-2 x$ & $x-2$ & 0 \\
{$[1,2]$} & $-x^{2}+5 x+1$ & $2 x-7$ & $2 x-7$ \\
{$[2,3]$} & $x^{2}-6 x+8$ & $x^{2}-6 x+8$ & 1 \\
{$[3,4]$} & 0 & $-x^{2}+x+2$ & $-x^{2}+x+2$ \\
{$[4,5]$} & $2 x-7$ & $x^{2}-2 x$ & $x-2$ \\
{$[5,5.5]$} & 1 & $x^{2}+15 x$ & $x^{2}+15 x$ \\
\hline
\end{tabular}

we change the $h$ value for a maximum reached value (let us call this new value $h_{\min }$ ) and move all facets so that they fill the area of $2 h_{\min }$. We use $h_{\min }$ just in this one step but for all facets and then get back to $h$ value. After each step, we recalculate domains and check whether we still use all functions (some of them may disappear, as the $x^{2}-2 x$ function defined on $[0,1]$ interval after the first step of the $v_{1}$ example from table 1).

In none of the presented examples, a facet fills the maximum area. We chose $h$ big enough to avoid unnecessary steps.

We calculate the time a step takes as $\frac{2 h_{\min }}{2 h}$. We do this using the following logic-we make an assumption that one full step (i.e., area of $2 h$ is filled) is our time unit, two full steps count as $t=2, \frac{1}{3} h$ takes $t=\frac{1}{3}$ to fill. In the pictures, accumulated time is presented.

As an initial data in three presented examples, we use functions described in the table below. The first column contains intervals which set the domain; the next three columns contain formulas for respective examples:

To create the three examples, we use the same domain and permute functions to obtain interesting shape. In some cases, we have to move parts defined on some intervals vertically to obtain continuous result. Therefore, in some cases, the same function used on the same interval has different values. What is more, we move the whole graph vertically so that the smallest value is 1 ; it makes integration easier without changing the shape of solutions.

We use polynomials as an approximation of a continuous function defined on closed interval; in the examples mentioned, they are of degree 2, but the algorithm remains the same for polynomials of higher degree. Functions defined on intervals model situation of noncontinuous derivative.

Let us look at results of simulations presented on the figures (Figs. 1, 2, 3):

Observe that all degenerated facets disappear after the first step of evolution. The number of regular facets that may appear is limited by their number, and the overall number of regular facets decreases from the second step of evolution. The flat area 

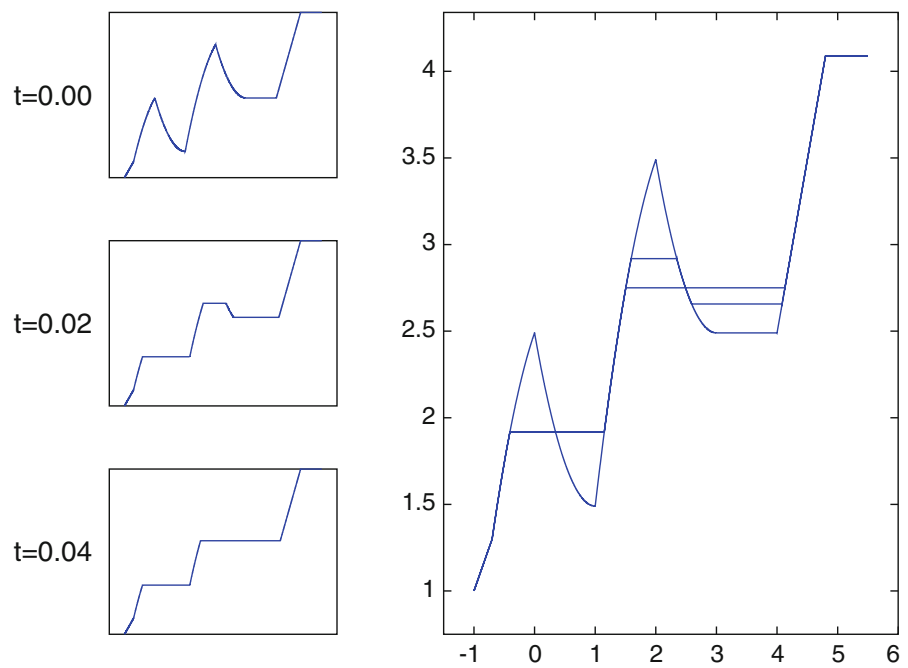

Figure 1. The first example
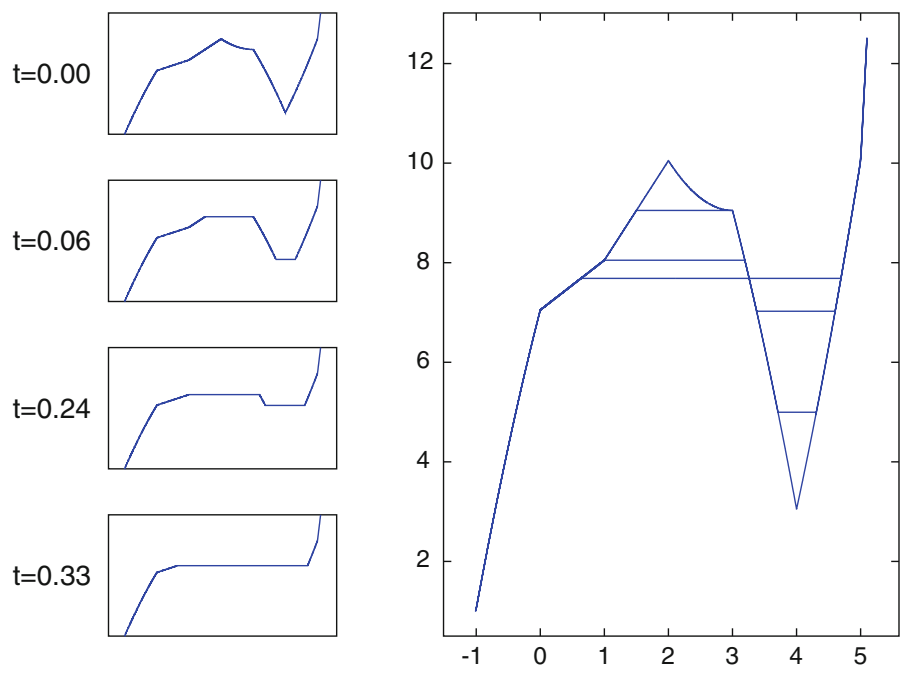

Figure 2. The second example

broadens with each step. All solutions remain continuous and their $\|\cdot\|_{L_{\infty}}$ norm is bounded by the norm of initial data.

We notice that similar looking computations were performed in [27] and [25]. However, they depended on a stability theorem, that is, the computations were done for a smoothed out anisotropy function. No specifics about technicalities of the solver were provided. 

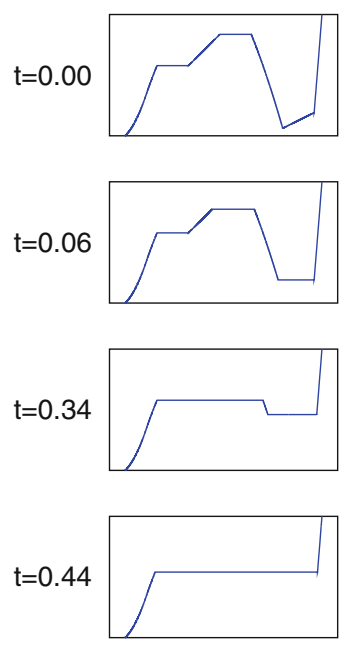

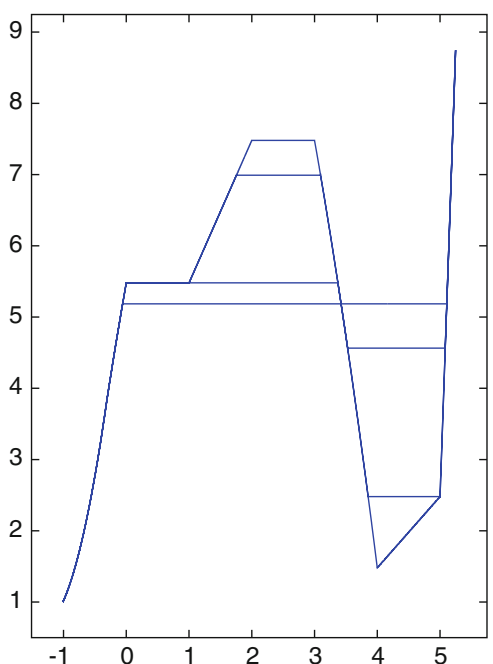

Figure 3. The third example

\section{Acknowledgments}

PR thanks Professor José Mazón for inspiring conversations on the topic of this paper. Both authors thank referees for precious comments improving the final version of the paper. In particular, one of the referees suggested the presented proof of Proposition 2.1. Special thanks go to the Iberia airline for creating extra opportunities to work on this paper and on related topics. After the submission of the paper, PR and PBM were informed by authors of [13] about their results concerning qualitative analysis around facets in 1d TVF. The present work has been partly supported by MN grant No. N N201 268935.

Open Access. This article is distributed under the terms of the Creative Commons Attribution License which permits any use, distribution, and reproduction in any medium, provided the original author(s) and the source are credited.

\section{REFERENCES}

[1] W.K. Allard, Total Variation Regularization for Image Denoising, I. Geometric theory, SIAM J. Math. Anal. 39 (2007/08), (4), 1150-1190.

[2] F. Andreu, C. Ballester, V. Caselles, J.M. Mazón, The Dirichlet problem for the total variation flow. J. Funct. Anal. 180, (2001), 347-403.

[3] F. Andreu, C. Ballester, V. Caselles, J.M. Mazón, Minimizing total variation flow. Diff. Int. Equations, 14, (2001), 321-360.

[4] S. Angenent, M.E. Gurtin, Multiphase thermomechanics with interfacial structure. II. Evolution of a isothermal interface. Arch. Rational Mech. Anal. 108, (1989), (4), 323-391.

[5] F. Andreu-Vaillo, V. Caselles, V., J.M. Mazón, "Parabolic quasilinear equations minimizing linear growth functionals". Birkhäuser, Basel, (2004).

[6] F. Andreu, V. Caselles, J.M. Mazón, S. Moll, The Dirichlet problem associated to the relativistic heat equation, Math. Ann., 347, (2010), 135-199. 
[7] F. Andreu, V. Caselles, J.I. Díaz, J.M. Mazón, Some qualitative properties for the total variation flow, J. Funct. Anal., 188, (2002), 516-547.

[8] G. Anzellotti, Pairings between measures and bounded functions and compensated compactness, Ann. Mat. Pura Appl. (4) 135 (1983), 293-318.

[9] H. Attouch, G. Buttazzo, G. Michaille, "Variational analysis in Sobolev and BV spaces. Applications to PDEs and optimization”. MPS/SIAM Series on Optimization 6. Philadelphia, PA, SIAM. Philadelphia, 2006.

[10] V. Barbu, "Nonlinear semigroups and differential equations in Banach spaces", Leyden, The Netherlands, Noordhoff International Publishing, 1976.

[11] P. Belik, M. Luskin, A total-variation surface energy model for thin films of martensitic crystals. Interfaces Free Bound., 4, (2002), 71-88.

[12] G. Bellettini, V. Caselles, M. Novaga, The total variation flow in $\mathbb{R}^{N}$. J. Diff. Eqns. , 184, (2002), 475-525

[13] M. Bonforte, A. Figalli, Total Variation Flow and Sign Fast Diffusion in one dimension, J. Differential Equations, 252, (2012) 4455-4480.

[14] A. Briani, A. Chambolle, M. Novaga, G. Orlandi On the gradient flow of a one-homogeneous functional, Confluentes Math., 3, (2011), 617-635.

[15] H. Brézis, "Opérateurs maximaux monotones et semi-groupes de contractions dans les espaces de Hilbert”, Amsterdam-London, North-Holland Publishing, 1973.

[16] K.-Ch. Chang, Variational methods for non-differentiable functionals and their applications to Partial Differential Equations, J. Math. Anal. Appl., 80, (1981), 102-129.

[17] M.G. Crandall, T.M. Liggett, Generation of semi-groups of nonlinear transformations on general Banach spaces. Amer. J. Math. 93, (1971), 265-298.

[18] G. diBlasio, Differtiability of Spatially Homogeneous Solution of the Boltzmann Equation in the Non Maxwellian Case. Commun. Math. Phys. 38, (1974), 331-340.

[19] X. Feng, M. Oehsen, A. Prohl, Rate of convergence of regularization procedures and finite element approximations for the total variation flow. Numer. Math., 100 (2005), (3), 441-456.

[20] T. Fukui, Y. Giga, Motion of a graph by nonsmooth weighted curvature, in: World Congress of Nonlinear Analysts '92, Vol. I-IV (Tampa, FL, 1992), 47-56, de Gruyter, Berlin, 1996.

[21] M.-H. Giga, Y. Giga, Very singular diffusion equations: second and fourth order problems, Japan J. Indust. Appl. Math., 27, (2010), 323-345.

[22] M.-H. Giga, Y. Giga, Evolving graphs by singular weighted curvature, Arch. Rational Mech. Anal. 141 (2) (1998), 117-198.

[23] M.-H. Giga, Y. Giga, Stability for evolving graphs by nonlocal weighted curvature, Comm. Partial Differential Equations, 24 (1-2) (1999), 109-184.

[24] M.-H. Giga, Y. Giga, Yoshikazu, H.Hontani, Self-similar expanding solutions in a sector for a crystalline flow, SIAM J. Math. Anal., 37 (4), (2006), 1207-1226.

[25] M.-H. Giga, Y. Giga, R. Kobayashi, Very Singular Diffusion Equations, Advanced Studies in Pure Mathematics 31, 2001 Taniguchi Conference on Mathematics Nara '98 93-125.

[26] M.-H. Giga, Y. Giga, P. Rybka, A comparison principle for singular diffusion equations with spatially inhomogeneous driving force, preprint.

[27] R. Kobayashi, Y. Giga, Equations with Singular Diffusivity, Journal of Statistical Physics, 95, (5/6), (1999), 1197-1220.

[28] P.B. Mucha, Global existence for the Einstein-Boltzmann equation in the flat Robertson-Walker spacetime. Comm. Math. Phys., 203 (1) (1999), 107-118.

[29] P.B. Mucha, P. Rybka, A new look at equilibria in Stefan type problems in the plane, SIAM J. Math. Anal. 39, (4), (2007), 1120-1134.

[30] P.B. Mucha, P. Rybka, A caricature of a singular curvature flow in the plane, Nonlinearity, 21, (2008), 2281-2316.

[31] P.B. Mucha, P. Rybka, Almost classical solutions of static Stefan type problems involving crystalline curvature, in: "Nonlocal and Abstract Parabolic Equations and their Applications", Banach Center Publ. 86, IMPAN, Warszawa, 2009, 223-234.

[32] J. Quah, D. Margetis, Anisotropic diffusion in continuum relaxation of stepped crystal surfaces, J. Phys. A: Math. Theor. 41 (2008) 235004 (18pp). 
[33] W. Ring, Structural properties of solutions to total variation regularization problems, M2AN Math. Model. Numer. Anal., 34 (4) (2000), 799-810.

[34] L.I. Rudin, S. Osher, E. Fatemi, Nonlinear total variation based noise removal argorithms, Physica $D, \mathbf{6 0},(1992), 259-268$.

[35] M. Struwe, "Variational Methods", Springer, New York, 1990.

[36] J. Taylor, Motion of curves by crystalline curvature, including triple junctions and boundary points, In: Differential Geometry: Partial Differential Equations on Manifolds (eds. R. Greene and S. T. Yau), Proc. Symp. Pure Math., 54 (1993) Part I, pp.417-438, Amer. Math. Society Providence, RI.

[37] Y.H.R. Tsai, Y. Giga, S. Osher, A level set approach for computing discontinuous solutions of Hamilton-Jacobi equations. Math. Comp.. 72 (241) (2003), 159-181.

[38] W.P. Ziemer, "Weakly differentiable functions", Springer, New York, 1989.

Karolina Kielak, Piotr Bogusław Mucha and Piotr Rybka

Instytut Matematyki Stosowanej i Mechaniki, Uniwersytet Warszawski,

ul. Banacha 2,

02-097 Warszawa,

Poland

E-mail:p.mucha@mimuw.edu.pl

Piotr Rybka

E-mail:p.rybka@mimuw.edu.pl 